\title{
Autoequivalences of derived categories of elliptic surfaces with non-zero Kodaira dimension
}

\author{
Hokuto Uehara \\ Dedicated to Professor Shigefumi Mori on the occasion of his retirement
}

\begin{abstract}
We study the group of autoequivalences of the derived category of coherent sheaves on a smooth projective elliptic surface with non-zero Kodaira dimension. We find a description of it when each reducible fiber is a cycle of $(-2)$-curves and non-multiple.
\end{abstract}

\section{Introduction}

\subsection{Motivation and results}

Let $X$ be a smooth projective variety over $\mathbb{C}$ and $D(X)$ the bounded derived category of coherent sheaves on $X$. If $X$ and $Y$ are smooth projective varieties with equivalent derived categories, then we call $X$ and $Y$ Fourier-Mukai partners. We define the set of isomorphism classes of FourierMukai partners of $X$ as

$$
\operatorname{FM}(X):=\{Y \text { smooth projective varieties } \mid D(X) \cong D(Y)\} / \cong .
$$

It is an interesting problem to determine the set $\operatorname{FM}(X)$ for a given $X$. There are several known results in this direction. For example, Bondal and Orlov show that if $K_{X}$ or $-K_{X}$ is ample, then $X$ can be entirely reconstructed from $D(X)$, namely $\operatorname{FM}(X)=\{X\}$ [BO97]. In contrast, there are examples of non-isomorphic varieties $X$ and $Y$ having equivalent derived categories. For example, in dimension 2, if $\operatorname{FM}(X) \neq\{X\}$, then $X$ is a K3 surface, an abelian surface or a relatively minimal elliptic surface with non-zero Kodaira dimension [BM01, Kaw02]. In dimension 3, Toda shows some results [Tod06]. Moreover, Orlov gives a complete answer to this problem for abelian varieties in [Orl02].

It is also natural to study the isomorphism classes of autoequivalences of $D(X)$. The group consisting of all exact $\mathbb{C}$-linear autoequivalences of $D(X)$ up to isomorphism is denoted by

$$
\text { Auteq } D(X) \text {. }
$$

We note that Auteq $D(X)$ always contains the group

$$
A(X):=\operatorname{Pic} X \rtimes \text { Aut } X \times \mathbb{Z}[1]
$$

Received 29 January 2015, accepted in final form 1 February 2016.

2010 Mathematics Subject Classification 14J27, 14F05, $18 \mathrm{E} 30$.

Keywords: derived category, elliptic surface, autoequivalence.

This journal is (C) Foundation Compositio Mathematica 2016. This article is distributed with Open Access under the terms of the Creative Commons Attribution Non-Commercial License, which permits non-commercial reuse, distribution, and reproduction in any medium, provided that the original work is properly cited. For commercial re-use, please contact the Foundation Compositio Mathematica.

The author is supported by the Grants-in-Aid for Scientific Research (No. 23340011). 


\section{H. UEHARA}

generated by the standard autoequivalences, namely the functors of tensoring with invertible sheaves, pull-backs along automorphisms and the shift functor [1].

When $K_{X}$ or $-K_{X}$ is ample, Bondal and Orlov show that Auteq $D(X) \cong A(X)$.

When $X$ is an abelian variety, Orlov determines the structure of Auteq $D(X)$ [Orl02]. As a special case, when $X$ is an elliptic curve, the autoequivalence group is described as

$$
1 \rightarrow \hat{X} \rtimes \text { Aut } X \times \mathbb{Z}[2] \rightarrow \text { Auteq } D(X) \stackrel{\theta}{\rightarrow} \mathrm{SL}(2, \mathbb{Z}) \rightarrow 1
$$

Here $\theta$ is given by the action of Auteq $D(X)$ on the even integral cohomology group $H^{0}(X, \mathbb{Z}) \oplus$ $H^{2}(X, \mathbb{Z})$, which is isomorphic to $\mathbb{Z}^{2}$. In this case, the group Auteq $D(X)$ contains the FourierMukai transform $\Phi_{J_{X}(a, b) \rightarrow X}^{\mathcal{U}}$ determined by a universal sheaf $\mathcal{U}$ of the fine moduli space $J_{X}(a, b)$ of stable vector bundles of rank $a$ and degree $b$ with $(a, b)=1$. By the work of Atiyah [Ati57, Theorem 7], the space $J_{X}(a, b)$ is isomorphic to $X$, and hence $\Phi_{J_{X}(a, b) \rightarrow X}^{\mathcal{U}}$ can be regarded as an autoequivalence of $D(X)$. One can check that $\Phi_{J_{X}(a, b) \rightarrow X}^{\mathcal{U}}$ does not belong to $A(X)$.

For the minimal resolution $X$ of $A_{n}$-singularities on a surface, Ishii and the author determine the structure of Auteq $D(X)$ in [IU05]. It is generated by the group $A(X)$ and twist functors of the form $T_{\mathcal{O}_{G}(a)}$ [ST01] associated with the line bundle $\mathcal{O}_{G}(a)$ on a $(-2)$-curve $G\left(\cong \mathbb{P}^{1}\right)$ on $X$. Again, $T_{\mathcal{O}_{G}(a)}$ does not belong to $A(X)$.

The case of smooth projective elliptic surfaces $\pi: S \rightarrow C$ with non-zero Kodaira dimension is a mixture of the last two cases. If $S$ has a reducible fiber, each component of it is a $(-2)$-curve. Hence Auteq $D(S)$ contains twist functors as in [IU05]. On the other hand, let us consider the fine moduli space $J_{S}(a, b)$ of pure 1-dimensional stable sheaves on $S$, the general point of which represents a rank $a$, degree $b$ stable vector bundle supported on a smooth fiber of $\pi$. It often occurs that there is an isomorphism $S \cong J_{S}(a, b)$, and then the Fourier-Mukai transform $\Phi_{J_{S}(a, b) \rightarrow S}^{\mathcal{U}}$ determined by a universal sheaf $\mathcal{U}$ on $J_{S}(a, b) \times S$ gives a non-standard autoequivalence.

For an object $E$ of $D(S)$, we define the fiber degree of $E$

$$
d(E)=c_{1}(E) \cdot F,
$$

where $F$ is a general fiber of $\pi$. Let us denote by $\lambda_{S}$ the greatest common factor of the fiber degrees of objects of $D(S)$. It is shown in [Bri98] that if $a \lambda_{S}$ and $b$ are coprime, the above-mentioned fine moduli space $J_{S}(a, b)$ exists. We write $J_{S}(b):=J_{S}(1, b)$.

We set

$$
\left.B:=\left\langle T_{\mathcal{O}_{G}(a)}\right| G \text { is a }(-2) \text {-curve }\right\rangle
$$

and denote the congruence subgroup of $\mathrm{SL}(2, \mathbb{Z})$ by

$$
\Gamma_{0}(m):=\left\{\left(\begin{array}{ll}
c & a \\
d & b
\end{array}\right) \in \mathrm{SL}(2, \mathbb{Z}) \mid d \in m \mathbb{Z}\right\}
$$

for $m \in \mathbb{Z}$.

Conjecture 1.1. Let $S$ be a smooth projective elliptic surface $S$ with $\kappa(S) \neq 0$. Then we have a short exact sequence

$$
\begin{aligned}
\left.1 \rightarrow\left\langle B, \otimes \mathcal{O}_{S}(D)\right| D \cdot F=0, F \text { is a fiber }\right\rangle & \rtimes \text { Aut } S \times \mathbb{Z}[2] \rightarrow \text { Auteq } D(S) \\
& \stackrel{\Theta}{\rightarrow}\left\{\left(\begin{array}{ll}
c & a \\
d & b
\end{array}\right) \in \Gamma_{0}\left(\lambda_{S}\right) \mid J_{S}(b) \cong S\right\} \rightarrow 1 .
\end{aligned}
$$

Here $\Theta$ is induced by the action of Auteq $D(S)$ on the even-degree part $H^{0}(F, \mathbb{Z}) \oplus H^{2}(F, \mathbb{Z}) \cong \mathbb{Z}^{2}$ of the integral cohomology group of a smooth fiber $F$. 


\section{Autoequivalences of Elliptic surfaces}

Remark 1.2. (i) As Conjecture 1.1 implicitly implies, we can actually see in the proof of Theorem 3.11 that every element of Auteq $D(S)$ induces an autoequivalence of a smooth fiber $F$. If $\kappa(S)=0$, this is false. See Example 2.8(iii).

(ii) The quotient group $\Gamma_{0}\left(\lambda_{S}\right) / \operatorname{Im} \Theta$ is naturally identified with the set of Fourier-Mukai partners $\operatorname{FM}(S)$ of $S$. See Remark 3.12.

(iii) If $\pi$ has a section, we know that $\operatorname{Im} \Theta \cong \operatorname{SL}(2, \mathbb{Z})$. See Remark 3.12.

(iv) By [IU05, Proposition 4.18], we have

$$
\left.\left.B \cap\left\langle\otimes \mathcal{O}_{S}(D)\right| D \cdot F=0, F \text { is a fiber }\right\rangle=\left\langle\otimes \mathcal{O}_{S}(G)\right| G \text { is a }(-2) \text {-curve }\right\rangle .
$$

The following is the main result of this article.

Theorem (Theorem 4.1). Suppose that each reducible fiber on the elliptic surface $S$ is nonmultiple and forms a cycle of (-2)-curves; that is, it is of type $\mathrm{I}_{n}$ for some $n>1$. Then Conjecture 1.1 is true.

In the case that $\pi$ has only irreducible fibers and $\pi$ has a section, Conjecture 1.1 is essentially shown in [LST13]. ${ }^{1}$

\subsection{Outline of the proof of Theorem 4.1}

Let $S$ be a projective elliptic surface with $\kappa(S) \neq 0$, and let $Z$ be the union of all reducible fibers of the elliptic fibration $\pi: S \rightarrow C$. Furthermore, let $U$ be the complement of $Z$ in $S$. We introduce a group homomorphism

$$
\iota_{U}: \text { Auteq } D(S) \rightarrow \text { Auteq } D(U)
$$

in Proposition 3.9 and denote $\operatorname{Im} \iota_{U}$ by Auteq $^{\dagger} D(U)$. We classify all elements of Auteq ${ }^{\dagger} D(U)$ in Proposition 3.6 and determine the structure of Auteq ${ }^{\dagger} D(U)$ in Theorem 3.11.

Assume furthermore that all reducible fibers of $\pi$ are of type $\mathrm{I}_{n}(n>1)$, as in Theorem 4.1. Then we can show that

$$
B=\operatorname{Ker} \iota_{U}
$$

by using the following result.

Proposition (Proposition 4.2). Take a connected component $Z_{0}$ of $Z$, that is, a fiber of $\pi$. Let us consider the irreducible decomposition $Z_{0}=C_{1} \cup \cdots \cup C_{n}$, where each $C_{i}$ is a (-2)-curve. Suppose that we are given an autoequivalence $\Phi$ of $D_{Z_{0}}(S)$ preserving the cohomology class $\operatorname{ch}\left(\mathcal{O}_{x}\right) \in H^{4}(S, \mathbb{Q})$ for some point $x \in Z_{0}$. Then, there are integers $a, b(1 \leqslant b \leqslant n)$ and $i$ and an autoequivalence

$$
\left.\Psi \in\left\langle T_{\mathcal{O}_{G}(a)}\right| G \text { is a }(-2) \text {-curves contained in } Z_{0}\right\rangle
$$

such that

$$
\Psi \circ \Phi\left(\mathcal{O}_{C_{1}}\right) \cong \mathcal{O}_{C_{b}}(a)[i]
$$

and

$$
\Psi \circ \Phi\left(\mathcal{O}_{C_{1}}(-1)\right) \cong \mathcal{O}_{C_{b}}(a-1)[i] .
$$

In particular, for any point $x \in C_{1}$, we can find a point $y \in C_{b}$ with $\Psi \circ \Phi\left(\mathcal{O}_{x}\right) \cong \mathcal{O}_{y}[i]$.

\footnotetext{
${ }^{1}$ The authors of [LST13] only consider autoequivalences $\Phi$ with $\Phi \in \operatorname{Ker} \delta$. See the definition of $\delta$ in Remark 3.10. On the other hand, their result is valid without any restrictions on the base space of the fibration.
} 


\section{H. UEHARA}

Proposition 4.2 is proved in Section 6 using techniques developed in [IU05]. Then we can deduce Theorem 4.1 from the equation (1.1) and the description of Auteq ${ }^{\dagger} D(U)$ obtained in Theorem 3.11.

The construction of this article is as follows. In Section 2, we show several preliminary results and give definitions needed afterwards. In Section 3, we study the structure of Auteq ${ }^{\dagger} D(U)$ intensively. In Section 4, we reduce the proof of Theorem 4.1 to showing equation (1.1), and furthermore we reduce the proof of (1.1) to showing Proposition 4.2. In Section 4, we also show several lemmas used in Sections 5 and 6. In Section 5, we show Proposition 4.4, which is the first step in the proof of Proposition 4.2. In Section 6, we prove Proposition 4.2. Finally, in Section 7, we treat an example of an elliptic surface satisfying the assumption of Theorem 4.1. In the example, we can determine the set $\operatorname{FM}(S)$, and also know when $J_{S}(b) \cong S$ holds. This information gives us a better description of $\operatorname{Im} \Theta$ (see Conjecture 1.1) in the example.

\subsection{Notation and conventions}

All varieties will be defined over $\mathbb{C}$. A point on a variety will always mean a closed point. By an elliptic surface, we will always mean a smooth surface $S$ together with a smooth curve $C$ and a relatively minimal projective morphism $\pi: S \rightarrow C$ whose general fiber is an elliptic curve. Here, a relatively minimal morphism means a morphism whose fibers contain no $(-1)$-curves.

For two elliptic surfaces $\pi: S \rightarrow C$ and $\pi^{\prime}: S^{\prime} \rightarrow C$, an isomorphism $\varphi: S \rightarrow S^{\prime}$ satisfying $\pi=\pi^{\prime} \circ \varphi$ is called an isomorphism over $C$.

For an elliptic curve $E$ and some positive integer $m$, we denote the set of points of order $m$ by ${ }_{m} E$. Furthermore, we denote the dual elliptic curve by $\hat{E}:=\mathrm{Pic}^{0} E$.

We denote by $D(X)$ the bounded derived category of coherent sheaves on an algebraic variety $X$. For a closed subset $Z$ of $X$, we denote the full subcategory of $D(X)$ consisting of objects supported on $Z$ by $D_{Z}(X)$. Here, the support of an object of $D(X)$ is, by definition, the union of the set-theoretic supports of its cohomology sheaves.

An object $\alpha$ in $D(X)$ is said to be simple (respectively, rigid) if

$$
\operatorname{Hom}_{D(X)}(\alpha, \alpha) \cong \mathbb{C} \quad\left(\text { respectively, } \operatorname{Hom}_{D(X)}^{1}(\alpha, \alpha) \cong 0\right) .
$$

Given a closed embedding of schemes $i: Z \hookrightarrow X$, we often denote the derived pull-back $\mathbb{L} i^{*} \alpha$ simply by $\left.\alpha\right|_{Z}$.

We denote by Auteq $\mathcal{T}$ the group of isomorphism classes of $\mathbb{C}$-linear exact autoequivalences of a $\mathbb{C}$-linear triangulated category $\mathcal{T}$.

\section{Preliminaries}

\subsection{General results for Fourier-Mukai transforms}

Let $X$ and $Y$ be smooth projective varieties. We call $Y$ a Fourier-Mukai partner of $X$ if $D(X)$ is $\mathbb{C}$-linear triangulated equivalent to $D(Y)$. We denote by $\operatorname{FM}(X)$ the set of isomorphism classes of Fourier-Mukai partners of $X$. be

For an object $\mathcal{P} \in D(X \times Y)$, we define the exact functor $\Phi^{\mathcal{P}}$, called an integral functor, to

$$
\Phi^{\mathcal{P}}:=\mathbb{R} p_{Y *}\left(\mathcal{P} \stackrel{\mathbb{L}}{\otimes} p_{X}^{*}(-)\right): D(X) \rightarrow D(Y),
$$

where we denote the projections by $p_{X}: X \times Y \rightarrow X$ and $p_{Y}: X \times Y \rightarrow Y$. We also sometimes write $\Phi^{\mathcal{P}}$ as $\Phi_{X \rightarrow Y}^{\mathcal{P}}$ to emphasize that it is a functor from $D(X)$ to $D(Y)$. 


\section{Autoequivalences of Elliptic surfaces}

Next, suppose that $X$ and $Y$ are not necessarily projective. Then, in general, $\mathbb{R} p_{Y *}$ is not well defined as a functor $D(X \times Y) \rightarrow D(Y)$ since $p_{Y}$ is not projective. Instead, suppose that there are projective morphisms $X \rightarrow C$ and $Y \rightarrow C$ over a smooth variety $C$, and let $\mathcal{P}$ be a perfect complex in $D\left(X \times{ }_{C} Y\right)$. Then we can also define the integral functor in this case, by replacing the projections $p_{Y}$ and $p_{X}$ with $p_{Y}: X \times_{C} Y \rightarrow Y$ and $p_{X}: X \times_{C} Y \rightarrow X$, respectively (note that we use the same notation for both kinds of projections). If we want to emphasize that we are in this situation, $\Phi$ is called a relative integral functor over $C$. Later, we use relative integral transforms in the case of elliptic surfaces over a non-projective base $C$.

By the result of Orlov [Or197], for smooth projective varieties $X$ and $Y$ and for a fully faithful functor $\Phi: D(X) \rightarrow D(Y)$, there is an object $\mathcal{P} \in D(X \times Y)$, unique up to isomorphism, such that

$$
\Phi \cong \Phi^{\mathcal{P}} .
$$

If an integral functor (over $C$ ) is an equivalence, it is called a Fourier-Mukai transform (over $C$ ).

The left adjoint to an integral functor $\Phi^{\mathcal{P}}$ over $C$ is given by the integral functor $\Phi^{\mathcal{Q}}$ over $C$, where

$$
\mathcal{Q}:=\mathbb{R} \mathcal{H} \operatorname{om}_{X \times_{C} Y}\left(\mathcal{P}, \mathcal{O}_{X \times_{C} Y}\right) \stackrel{\mathbb{L}}{\otimes} p_{X}^{*} \omega_{X / C}[\operatorname{dim} X-\operatorname{dim} C]
$$

(see the proof of [Huy06, Proposition 5.9]). In particular, if $\Phi^{\mathcal{P}}$ is an equivalence, its quasi-inverse is given by $\Phi^{\mathcal{Q}}$.

We can also see that the composition of integral functors over $C$ is again an integral functor over $C$ (cf. [Huy06, Proposition 5.10]).

Lemma 2.1. Let $\Phi: D(X) \rightarrow D(Y)$ be a Fourier-Mukai transform over a smooth variety $C$ between smooth varieties $X$ and $Y$ that are projective over $C$. Then the set of points $x \in X$ for which the object $\Phi\left(\mathcal{O}_{x}\right)$ is a sheaf forms a (possibly empty) open subset of $X$.

Proof. This is a special case of [BM01, Proposition 2.4]. See also the proof of [BM01, Lemma 2.5].

The following is well known.

Lemma 2.2. Let $\Phi: D(X) \rightarrow D(Y)$ be a Fourier-Mukai transform over a smooth variety $C$ between smooth varieties $X$ and $Y$ that are projective over $C$. Assume that $\Phi\left(\mathcal{O}_{x}\right)$ is a shift of a sheaf supported on a finite subset of $Y$ for all points $x \in X$. Then we have

$$
\Phi \cong \phi_{*} \circ((-) \otimes \mathcal{L})[n]
$$

for a line bundle $\mathcal{L}$ on $X$, an isomorphism $\phi: X \rightarrow Y$ over $C$ and some integer $n$.

Proof. By the assumptions, $\Phi\left(\mathcal{O}_{x}\right)$ satisfies the condition of [Huy06, Lemma 4.5]. Hence, $\Phi\left(\mathcal{O}_{x}\right) \cong$ $\mathcal{O}_{y}[n]$ for some $y \in Y$ and $n \in \mathbb{Z}$. Note that the integer $n$ does not depend on the choice of a point $x$, by Lemma 2.1. Then apply [BM98, Section 3.3] (or [Huy06, Corollary 5.23]) to get the conclusion.

Lemma 2.3. Let $X$ be a smooth variety, $E \in D(X)$ an object with compact support and $x \in X$. Then $\mathbb{R H o m}_{D(X)}\left(E, \mathcal{O}_{x}\right) \neq 0$ holds if and only if $x \in \operatorname{Supp} E$. Moreover, these conditions are also equivalent to $\mathbb{R H o m}_{D(X)}\left(\mathcal{O}_{x}, E\right) \neq 0$.

Proof. The first statement is just [BM02, Lemma 5.3]. The second follows from Serre duality and the first. 


\section{H. UEHARA}

Lemma 2.4. Let $X$ and $Y$ be smooth projective varieties given together with closed subsets $Z \subset X$ and $W \subset Y$. Suppose that a Fourier-Mukai transform $\Phi=\Phi_{X \rightarrow Y}$ and its quasi-inverse $\Psi$ satisfy

$$
\operatorname{Supp} \Phi\left(\mathcal{O}_{z}\right) \subset W \text { and } \operatorname{Supp} \Psi\left(\mathcal{O}_{w}\right) \subset Z
$$

for all points $z \in Z$ and $w \in W$. Then $\Phi$ restricts to a Fourier-Mukai transform from $D_{Z}(X)$ to $D_{W}(Y)$.

Proof. We repeatedly use Lemma 2.3. Take points $z \in Z$ and $y \in Y \backslash W$. Then

$$
\mathbb{R H o m}_{D(X)}\left(\Psi\left(\mathcal{O}_{y}\right), \mathcal{O}_{z}\right)=\mathbb{R} \operatorname{Hom}_{D(Y)}\left(\mathcal{O}_{y}, \Phi\left(\mathcal{O}_{z}\right)\right)
$$

vanishes by the assumption. This means that $\operatorname{Supp} \Psi\left(\mathcal{O}_{y}\right) \cap Z=\emptyset$, and thus for an object $E \in D_{Z}(X)$, we have

$$
\mathbb{R H o m}_{D(Y)}\left(\Phi(E), \mathcal{O}_{y}\right)=\mathbb{R} \operatorname{Hom}_{D(X)}\left(E, \Psi\left(\mathcal{O}_{y}\right)\right)=0,
$$

which implies that $\Phi(E) \in D_{W}(Y)$. Here, the last equality follows from [Huy06, Lemma 3.9]. In a similar way, we can prove that $\Psi(F) \in D_{Z}(X)$ for any object $F \in D_{W}(Y)$. Therefore, we obtain the conclusion.

The following is also well known.

Lemma 2.5 (cf. [HLS09, Proposition 2.15]). Let $\pi: X \rightarrow C$ and $\pi^{\prime}: Y \rightarrow C$ be flat projective morphisms between smooth varieties, and take a point $c$ of $C$. Suppose that $X_{c}$ and $Y_{c}$ are the fibers of $\pi$ and $\pi^{\prime}$, respectively, over the point $c$, and that we are given an integral functor $\Phi=\Phi_{X \rightarrow Y}^{\mathcal{P}}$ over $C$.

Let us consider the integral functor $\Psi=\Phi_{X_{c} \rightarrow Y_{c}}^{\left.\mathcal{P}\right|_{X_{c} \times Y_{c}}}$, and denote the inclusions by $k: X_{c} \hookrightarrow X$ and $k^{\prime}: Y_{c} \hookrightarrow Y$. (Although we do not assume that $X_{c}$ and $Y_{c}$ are smooth, the perfectness of $\left.\mathcal{P}\right|_{X_{c} \times Y_{c}}$ assures that $\Psi$ defines a functor from $D\left(X_{c}\right)$ to $D\left(Y_{c}\right)$.) Then we have the following:

(i) The functors $\Phi$ and $\Psi$ satisfy $k_{*}^{\prime} \circ \Psi \cong \Phi \circ k_{*}$.

(ii) Assume furthermore that $\Phi$ is a Fourier-Mukai transform. Then so is $\Psi$.

Proof. Assertion (i) follows directly from the projection formula and the flat base change formula (cf. [Huy06, Section 3.3, pp. 83, 85]). For assertion (ii), suppose $X=Y$ and $\Phi \cong \mathrm{id}_{X}$. Then obviously $\Psi \cong \mathrm{id}_{X_{c}}$ also holds. Apply this argument to the functor $\Phi \circ \Phi^{-1}$ to get the result.

\subsection{The Euler form on surfaces}

Let $X$ be a smooth quasi-projective variety. For objects $\alpha, \beta \in D(X)$ with compact supports, we define the Euler form as

$$
\chi(\alpha, \beta):=\sum_{i}(-1)^{i} \operatorname{dim} \operatorname{Hom}_{D(X)}^{i}(\alpha, \beta) .
$$

In the surface case, the Riemann-Roch theorem yields

$$
\begin{aligned}
\chi(\alpha, \beta)= & r(\alpha) \operatorname{ch}_{2}(\beta)-c_{1}(\alpha) \cdot c_{1}(\beta)+r(\beta) \operatorname{ch}_{2}(\alpha) \\
& +\frac{1}{2}\left(r(\beta) c_{1}(\alpha)-r(\alpha) c_{1}(\beta)\right) \cdot c_{1}\left(\omega_{X}\right)+r(\alpha) r(\beta) \chi\left(\mathcal{O}_{X}\right) .
\end{aligned}
$$

In particular, if $r(\alpha)=r(\beta)=0$, we have

$$
\chi(\alpha, \beta)=-c_{1}(\alpha) \cdot c_{1}(\beta) .
$$




\section{Autoequivalences of Elliptic surfaces}

As an application, for a (-2)-curve $G$ on a smooth surface $X$, we can compute

$$
\operatorname{dim} \operatorname{Ext}_{X}^{1}\left(\mathcal{O}_{G}(a), \mathcal{O}_{G}(b)\right)= \begin{cases}b-a-1 & \text { if } b-a>1 \\ 0 & \text { if }|b-a| \leqslant 1 \\ a-b-1 & \text { if } a-b>1\end{cases}
$$

\subsection{Twist functors}

We introduce an important class of examples of autoequivalences.

Definition-PRoposition 2.6 ([ST01]). Let $X$ be a smooth variety, or rather a complex manifold.

(i) We say that an object $\alpha \in D(X)$ with compact support is spherical if we have $\alpha \otimes \omega_{X} \cong \alpha$ and

$$
\operatorname{Hom}_{D(X)}^{k}(\alpha, \alpha) \cong \begin{cases}0, & k \neq 0, \operatorname{dim} X, \\ \mathbb{C}, & k=0, \operatorname{dim} X\end{cases}
$$

(ii) Let $\alpha \in D(X)$ be a spherical object. We consider the mapping cone

$$
\mathcal{C}=\operatorname{Cone}\left(\pi_{1}^{*} \alpha^{\vee} \stackrel{\mathbb{L}}{\otimes} \pi_{2}^{*} \alpha \rightarrow \mathcal{O}_{\Delta}\right)
$$

of the natural evaluation $\pi_{1}^{*} \alpha^{\vee} \stackrel{\mathbb{L}}{\otimes} \pi_{2}^{*} \alpha \rightarrow \mathcal{O}_{\Delta}$, where $\Delta \subset X \times X$ is the diagonal and $\pi_{i}$ is the projection of $X \times X$ to the $i$ th factor. Then the integral functor $T_{\alpha}:=\Phi_{X \rightarrow X}^{\mathcal{C}}$ defines an autoequivalence of the bounded derived category $D_{\mathrm{Coh}(X)}\left(\mathcal{O}_{X}-\bmod \right)$ of $\mathcal{O}_{X}$-modules with coherent cohomology on $X$, called the twist functor along the spherical object $\alpha$. (For an algebraic variety $X$ or a compact complex analytic space $X$ of dimension 2 , it is known that $D_{\operatorname{Coh}(X)}\left(\mathcal{O}_{X}-\bmod \right)$ is equivalent to $D(X)$. See [Huy06, Corollary 3.4, Proposition 3.5] and [BV03, Corollary 5.2.2].)

Remark 2.7. Let $\alpha \in D(X)$ be a spherical object. Then, by definition, for every $\beta \in D(X)$, we have an exact triangle

$$
\mathbb{R H o m}_{D(X)}(\alpha, \beta) \otimes_{\mathbb{C}} \alpha \rightarrow \beta \rightarrow T_{\alpha}(\beta) .
$$

In particular, in the Grothendieck group $K(X)$, we have

$$
\left[T_{\alpha}(\beta)\right]=[\beta]-\chi(\alpha, \beta)[\alpha] .
$$

Suppose $\operatorname{Supp} \alpha \cap \operatorname{Supp} \beta=\emptyset$. Then since $\mathbb{R} \operatorname{Hom}(\alpha, \beta)=0$, we have $T_{\alpha}(\beta) \cong \beta$. We use this remark later.

Example 2.8. (i) Let $S$ be a smooth surface, and let $G$ be a $(-2)$-curve. Then, for every integer $a$ and any point $x \in S$, we can deduce

$$
\chi\left(\mathcal{O}_{G}(a), \mathcal{O}_{G}(a)\right)=2 \quad \text { and } \quad \chi\left(\mathcal{O}_{G}(a), \mathcal{O}_{x}\right)=0
$$

by equality (2.1). Hence, the object $\mathcal{O}_{G}(a) \in D(S)$ is spherical, and it follows from equality (2.4) that $\left[T_{\mathcal{O}_{G}(a)}\left(\mathcal{O}_{x}\right)\right]=\left[\mathcal{O}_{x}\right]$. By using $(2.3)$, we can also see that

$$
H^{-1}\left(T_{\mathcal{O}_{G}}\left(\mathcal{O}_{G}(2)\right)\right)=\mathcal{O}_{G}(-1)^{\oplus 2} \text { and } H^{0}\left(T_{\mathcal{O}_{G}}\left(\mathcal{O}_{G}(2)\right)\right)=\mathcal{O}_{G},
$$

and hence $T_{\mathcal{O}_{G}} \notin A(S)$.

(ii) Let $\alpha$ be a simple coherent sheaf on an elliptic curve $E$, for example a line bundle or the structure sheaf $\mathcal{O}_{x}$ of a point $x \in E$. Then, $\alpha$ is spherical. Usually, twist functors are not standard autoequivalences, but we can see that in this case $T_{\mathcal{O}_{x}} \cong \otimes \mathcal{O}_{E}(x) \in A(E)$ (cf. [Huy06, Example 8.10]). 


\section{H. UEHARA}

(iii) Let $X$ be a K3 surface. Then, the structure sheaf $\mathcal{O}_{X}$ of $X$ is spherical. We can see that $\operatorname{Supp} T_{\mathcal{O}_{X}}\left(\mathcal{O}_{x}\right)=2$ by using the triangle (2.3). In contrast, we will see in Claim 3.1 that for an elliptic surface $S$ with non-zero Kodaira dimension, any autoequivalence $\Phi \in$ Auteq $D(S)$ satisfies $\operatorname{Supp} \Phi\left(\mathcal{O}_{x}\right) \leqslant 1$.

Theorem 2.9 ([IU05, Theorem 1.3], [IUU10, Appendix A]). Let $X$ be a minimal resolution of the $A_{n}$-singularity Spec $\mathbb{C}[[x, y, z]] /\left(x^{2}+y^{2}+z^{n+1}\right)$. Define

$$
\left.B:=\left\langle T_{\mathcal{O}_{G}(a)}\right| G \text { is a }(-2) \text {-curve }\right\rangle,
$$

and denote by $Z$ the exceptional set of the resolution. Then we have

$$
\text { Auteq } D_{Z}(X)=(\langle B, \operatorname{Pic} X\rangle \rtimes \operatorname{Aut} X) \times \mathbb{Z} .
$$

We will use ideas of the proof of Theorem 2.9 to prove our main result, Theorem 4.1.

\subsection{Autoequivalences of elliptic curves}

Let $E$ be an elliptic curve. With a given $\Phi=\Phi^{\mathcal{P}} \in$ Auteq $D(E)$, we associate a group automorphism $\rho(\Phi)$ of $H^{*}(E, \mathbb{Z}) \cong \mathbb{Z}^{4}$ given by

$$
\rho\left(\Phi^{\mathcal{P}}\right)(-):=p_{2 *}\left(\operatorname{ch}(\mathcal{P}) \cdot p_{1}^{*}(-)\right)
$$

(cf. [Huy06, Corollary 9.43]). This gives a group homomorphism

$$
\rho: \quad \operatorname{Auteq} D(E) \rightarrow \operatorname{GL}\left(H^{*}(E, \mathbb{Z})\right),
$$

and it is known that $\rho$ preserves the parity; that is, it decomposes as $\rho=\eta \oplus \theta$, where $\eta(\Phi) \in$ $\mathrm{GL}\left(H^{\text {odd }}(E, \mathbb{Z})\right)$ and $\theta(\Phi) \in \mathrm{GL}\left(H^{\mathrm{ev}}(E, \mathbb{Z})\right)$.

Because $\theta(\Phi) \in \mathrm{GL}(2, \mathbb{Z})$ preserves the Euler form $\chi\left({ }_{-},-\right)$, we can see that $\theta(\Phi)$ actually gives an element of $\mathrm{SL}(2, \mathbb{Z})$. Take the classes $\operatorname{ch}\left(\mathcal{O}_{E}\right)$ and $\operatorname{ch}\left(\mathcal{O}_{x}\right)$ for some point $x$ as a basis of $H^{\mathrm{ev}}(E, \mathbb{Z}) \cong \mathbb{Z}^{2}$. In terms of this basis, $T_{\mathcal{O}_{E}}, T_{\mathcal{O}_{x}}\left(\cong{ }_{-} \otimes \mathcal{O}_{E}(x)\right)$ and $\Phi^{\mathcal{U}}$ are given by

$$
\left(\begin{array}{cc}
1 & -1 \\
0 & 1
\end{array}\right), \quad\left(\begin{array}{ll}
1 & 0 \\
1 & 1
\end{array}\right), \quad\left(\begin{array}{cc}
0 & 1 \\
-1 & 0
\end{array}\right)
$$

respectively, where $\mathcal{U}$ is the normalized Poincaré bundle on $E \times E$. Here, note that every elliptic curve is principally polarized, and hence we can identify $E$ with $\hat{E}$. Two of these elements actually generate the group $\operatorname{SL}(2, \mathbb{Z})$, and therefore the map

$$
\theta: \text { Auteq } D(E) \rightarrow \mathrm{SL}(2, \mathbb{Z})
$$

is surjective. One can compute the kernel of $\theta$ to get a short exact sequence of groups

$$
1 \rightarrow \hat{E} \rtimes \text { Aut } E \times \mathbb{Z}[2] \rightarrow \text { Auteq } D(E) \rightarrow \mathrm{SL}(2, \mathbb{Z}) \rightarrow 1 .
$$

\subsection{Automorphisms of elliptic surfaces}

Let $\pi: S \rightarrow C$ and $\pi^{\prime}: S^{\prime} \rightarrow C^{\prime}$ be projective elliptic surfaces, and suppose that $S$ and $S^{\prime}$ each have a unique elliptic fibration. Then every isomorphism $\varphi: S \rightarrow S^{\prime}$ induces an isomorphism $C \rightarrow C^{\prime}$. In the cases that we consider (namely that $S^{\prime}=J_{S}(a, b)$; see Section 2.6) there is a natural identification between $C$ and $C^{\prime}$. Hence, the induced isomorphism is naturally regarded as an automorphism of $C$. We denote it by $\varphi_{C}$. In other words, $\varphi_{C}$ satisfies $\pi^{\prime} \circ \varphi=\varphi_{C} \circ \pi$. We define

$$
\operatorname{Aut}_{S} C:=\left\{\varphi_{C} \in \operatorname{Aut} C \mid \varphi \in \operatorname{Aut} S\right\}
$$




\section{Autoequivalences of Elliptic surfaces}

and

$$
\operatorname{Aut}_{C} S:=\left\{\varphi \in \text { Aut } S \mid \varphi_{C}=\operatorname{id}_{C}\right\} .
$$

Consequently, we have a short exact sequence

$$
1 \rightarrow \operatorname{Aut}_{C} S \rightarrow \text { Aut } S \rightarrow \operatorname{Aut}_{S} C \rightarrow 1
$$

An elliptic surface $S$ with $\kappa(S) \neq 0$ provides an example of a surface admitting a unique elliptic fibration: the canonical bundle formula of elliptic surfaces implies that any elliptic fibration on $S$ is defined by the linear system $\left|r K_{S}\right|$ with some non-zero rational number $r$; compare with [Bea96, Proposition IX.3].

\subsection{Fourier-Mukai transforms on elliptic surfaces}

Bridgeland, Maciocia and Kawamata show [BM01, Kaw02] that for a smooth projective surface $S$, if $S$ has a non-trivial Fourier-Mukai partner $T$, that is, $|\operatorname{FM}(S)| \neq 1$, then both $S$ and $T$ are abelian varieties, K3 surfaces or minimal elliptic surfaces with non-zero Kodaira dimensions.

We consider the last case in more detail. Let $\pi: S \rightarrow C$ be an elliptic surface. The results referred to in this section are originally stated under the assumption that $S$ is projective, but some of them still hold true without the projectivity of $S$. For our purpose, it is sometimes important to consider non-projective elliptic surfaces, hence we do not assume that $S$ is projective unless specified otherwise.

For an object $E$ of $D(S)$, we define the fiber degree of $E$ as

$$
d(E)=c_{1}(E) \cdot F,
$$

where $F$ is a general fiber of $\pi$. Let us denote by $r(E)$ the rank of $E$ and by $\lambda_{S}$ the greatest common factor of the fiber degrees of the objects of $D(S)$. Equivalently, $\lambda_{S}$ is the smallest number $d$ such that there is a holomorphic $d$-section of $\pi$. Consider integers $a$ and $b$ with $a>0$ and $b$ coprime to $a \lambda_{S}$. By [Bri98], there exists a smooth, 2-dimensional component $J_{S}(a, b)$ of the moduli space of pure dimension 1 stable sheaves on $S$, the general point of which represents a rank $a$, degree $b$ stable vector bundle supported on a smooth fiber of $\pi$. There is a natural morphism $J_{S}(a, b) \rightarrow C$, taking a point representing a sheaf supported on the fiber $\pi^{-1}(x)$ of $S$ to the point $x$. This morphism is a minimal elliptic fibration (see [Bri98]). Put $J_{S}(b):=J_{S}(1, b)$. Obviously, $J_{S}(0)$ is isomorphic to $J(S)$, the Jacobian surface associated with $S$, and $J_{S}(1) \cong S$. As shown in [BM01, Lemma 4.2], there is also an isomorphism

$$
J_{S}(a, b) \cong J_{S}(b)
$$

over $C$.

Theorem 2.10 ([Bri98, Theorem 5.3]). Let $\pi: S \rightarrow C$ be an elliptic surface, and take an element

$$
M=\left(\begin{array}{ll}
c & a \\
d & b
\end{array}\right) \in \mathrm{SL}(2, \mathbb{Z})
$$

such that $\lambda_{S}$ divides $d$ and $a>0$. Then there exists a universal sheaf $\mathcal{U}$ on $J_{S}(a, b) \times S$, flat over both factors, such that for any point $(x, y) \in J_{S}(a, b) \times S$, the restriction $\left.\mathcal{U}\right|_{x \times S}$ has Chern class $(0, a f,-b)$ on $S$ and $\left.\mathcal{U}\right|_{J_{S}(a, b) \times y}$ has Chern class $(0, a f,-c)$ on $J_{S}(a, b)$. The resulting functor $\Phi_{J_{S}(a, b) \rightarrow S}^{\mathcal{U}}$ is an equivalence and satisfies

$$
\left(\begin{array}{l}
r(\Phi(E)) \\
d(\Phi(E))
\end{array}\right)=M\left(\begin{array}{l}
r(E) \\
d(E)
\end{array}\right)
$$




\section{H. UEHARA}

for all objects $E \in D\left(J_{S}(a, b)\right)$

Remark 2.11. For integers $a>0$ and $b$ with $b$ coprime to $a \lambda_{S}$, let us consider the FourierMukai transform $\Phi=\Phi_{J_{S}(a, b) \rightarrow S}^{\mathcal{U}}$. Take a smooth fiber $F$ of $\pi$ over a point $x \in C$, and denote by $F^{\prime}$ the smooth fiber of the morphism $J_{S}(a, b) \rightarrow C$ over the point $x$. It turns out that $F^{\prime}$ is isomorphic to $J_{F}(a, b)$, and hence $F^{\prime}$ is isomorphic to $F$ by [Ati57, Theorem 7]. The integral transform defined by the kernel $\left.\mathcal{U}\right|_{F \times F^{\prime}} \in D\left(F \times F^{\prime}\right)$ induces an equivalence between $D(F)$ and $D\left(F^{\prime}\right)$ by Lemma 2.5. Fixing an isomorphism $F \cong F^{\prime}$, we regard the equivalence $\Phi^{\left.\mathcal{U}\right|_{F \times F^{\prime}}}$ as an autoequivalence of $D(F)$.

Note that

$$
M:=\theta\left(\Phi^{\left.\mathcal{U}\right|_{F \times F^{\prime}}}\right):=\left(\begin{array}{ll}
c & a \\
d & b
\end{array}\right)
$$

satisfies (2.6) (see Section 2.4 for the definition of $\theta$ ). We see that $\lambda_{S}$ divides $d=d\left(\Phi\left(\mathcal{O}_{J_{S}(a, b)}\right)\right.$ ). We will use this fact in Section 3.4.

Theorem 2.10 implies that $J_{S}(b)\left(\cong J_{S}(a, b)\right)$ is a Fourier-Mukai partner of $S$ when $\left(b, \lambda_{S}\right)=1$. Actually, the converse is also true for projective elliptic surfaces $S$ with non-zero Kodaira dimension.

Theorem 2.12 ([BM01, Proposition 4.4]). Let $\pi: S \rightarrow C$ be a projective elliptic surface and $S^{\prime}$ a smooth projective variety. Assume that the Kodaira dimension $\kappa(S)$ is non-zero. Then the following are equivalent:

(i) The variety $S^{\prime}$ is a Fourier-Mukai partner of $S$.

(ii) The variety $S^{\prime}$ is isomorphic to $J_{S}(b)$ for some integer $b$ with $\left(b, \lambda_{S}\right)=1$.

Remark 2.13. Theorem 2.12 tells us that the Fourier-Mukai partner $S^{\prime}$ of $S$ has an elliptic fibration $\pi^{\prime}: S^{\prime} \rightarrow C$. Moreover, we can see that $\kappa\left(S^{\prime}\right)=\kappa(S) \neq 0$ (cf. [BM01, Lemma 4.3]). Then, as is explained in Section 2.5, the morphism $\pi^{\prime}$ is a unique elliptic fibration structure on $S^{\prime}$. In particular, if two elliptic surfaces are mutually Fourier-Mukai partners, the base curves of the elliptic fibrations can be identified. We use this fact implicitly later.

There are natural isomorphisms

$$
J_{S}(b) \cong J_{S}\left(b+\lambda_{S}\right) \cong J_{S}(-b)
$$

over $C$ (see [BM01, Remark 4.5]). Therefore, we can define the subset

$$
H_{S}:=\left\{b \in\left(\mathbb{Z} / \lambda_{S} \mathbb{Z}\right)^{*} \mid J_{S}(b) \cong S\right\}
$$

of the multiplicative group $\left(\mathbb{Z} / \lambda_{S} \mathbb{Z}\right)^{*}$.

Claim 2.14. For any pair of integers $b, c$, with $b, c \in \mathbb{Z} / \lambda_{S} \mathbb{Z}$, there is an isomorphism

$$
J_{J_{S}(c)}(b) \cong J_{S}(b c) \text {. }
$$

Proof. Take an elliptic surface $B \rightarrow C$ with a section such that there is an isomorphism $\varphi_{1}: J(S) \rightarrow B$ over $C$. Let us set $\xi:=\left(S, \varphi_{1}\right) \in \mathrm{WC}(B)$ (see [Ueh12, Section 2.2] for the definition of the Weil-Chatelet group $\mathrm{WC}(B))$. Then we see in [Ueh12, Section 2.2] that there is an isomorphism $\varphi_{c}: J\left(J_{S}(c)\right) \rightarrow B$ over $C$ such that $\left(J_{S}(c), \varphi_{c}\right)$ corresponds to the element $c \xi \in \mathrm{WC}(B)$. By the same argument, there are isomorphisms $\varphi_{b c}^{\prime}: J\left(J_{J_{S}(c)}(b)\right) \rightarrow B$ and $\varphi_{b c}: J\left(J_{S}(b c)\right) \rightarrow B$ such that both $\left(J_{J_{S}(c)}(b), \varphi_{b c}^{\prime}\right)$ and $\left(J_{S}(b c), \varphi_{b c}\right)$ correspond to the element $b c \xi$. This finishes the proof. 


\section{Autoequivalences of Elliptic surfaces}

Since we have $J_{S}(1) \cong S$ (see, for example, [Ueh12, Section 2.2] and [BM01, Remark 4.5]), we can see that the condition $J_{S}(b) \cong S$ implies $J_{S}(c) \cong S$ for $c \in \mathbb{Z}$ with $b c \equiv 1(\bmod m)$. Therefore, it turns out that $H_{S}$ is a subgroup of $\left(\mathbb{Z} / \lambda_{S} \mathbb{Z}\right)^{*}$. In particular, there is a natural one-to-one correspondence between the set $\operatorname{FM}(S)$ and the quotient group $\left(\mathbb{Z} / \lambda_{S} \mathbb{Z}\right)^{*} / H_{S}$.

It is not easy to describe the group $H_{S}$ concretely in general, or, equivalently, to determine the set $\operatorname{FM}(S)$ (see [Ueh04] and [Ueh12]). However, when $\lambda_{S} \leqslant 2$, the group $\left(\mathbb{Z} / \lambda_{S} \mathbb{Z}\right)^{*}$ is trivial, and hence $\operatorname{FM}(S)=\{S\}$.

If $\lambda_{S}>2$, the group $H_{S}$ contains at least two elements $1, \lambda_{S}-1 \in\left(\mathbb{Z} / \lambda_{S} \mathbb{Z}\right)^{*}$. Hence, we have

$$
|\operatorname{FM}(S)| \leqslant \varphi\left(\lambda_{S}\right) / 2,
$$

where $\varphi$ is the Euler function. Several examples in which we can compute the set $\operatorname{FM}(S)$ are given in [Ueh12, Example 2.6]. In [Ueh15], the author provides further examples in which he can compute the set $\operatorname{FM}(S)$.

Remark 2.15. Take a point $c \in C$ and an integer $b$ with $\left(b, \lambda_{S}\right)=1$. We know that there is an isomorphism $J\left(J_{S}(b)\right) \cong J(S)$ over the curve $C$ (cf. [Ueh12, Section 2.2]). Since it is known that the reduced form ${ }^{2}$ of the fibers of $S$ over the point $c$ is isomorphic to the fiber of $J(S)$ over $c$, the same holds for the fibers of $S$ and $J_{S}(b)$.

Furthermore, the multiplicities of the fibers of $S$ and $J_{S}(b)$ over the same point are equal (see [Fri95, Part I, Section 1, p. 38] or the proof of [BM01, Lemma 4.3]). Therefore, we conclude that the fibers of $S$ and $J_{S}(b)$ over the same point are isomorphic to each other.

\section{Autoequivalences of elliptic surfaces with non-zero Kodaira dimension}

\subsection{Notation and setting}

Let

$$
\pi: S \rightarrow C \text { and } \pi^{\prime}: S^{\prime} \rightarrow C
$$

be projective elliptic surfaces with non-zero Kodaira dimension, and denote the projections by

$$
p: S \times S^{\prime} \rightarrow S \text { and } p^{\prime}: S \times S^{\prime} \rightarrow S^{\prime} .
$$

Let $\Phi=\Phi^{\mathcal{P}}: D(S) \rightarrow D\left(S^{\prime}\right)$ be a Fourier-Mukai transform. The following is well known.

Claim 3.1. For each $y \in S$, the support $\operatorname{Supp} \Phi\left(\mathcal{O}_{y}\right)$ is contained in a single fiber of $\pi^{\prime}$. Furthermore, for a point $x \in S$ with $\pi(x) \neq \pi(y)$, we have $\operatorname{Supp} \Phi\left(\mathcal{O}_{y}\right) \cap \operatorname{Supp} \Phi\left(\mathcal{O}_{x}\right)=\emptyset$.

Proof. By the assumption on the Kodaira dimension, the cohomology class of $K_{S^{\prime}}$ is a non-zero rational multiple of the cohomology class of a fiber of $\pi^{\prime}$. On the other hand, since the Serre functor commutes with the equivalence $\Phi$, there is an isomorphism

$$
\Phi\left(\mathcal{O}_{y}\right) \cong \Phi\left(\mathcal{O}_{y}\right) \otimes \omega_{S^{\prime}} .
$$

Furthermore, since $\Phi\left(\mathcal{O}_{y}\right)$ is simple, we know that $\operatorname{Supp} \Phi\left(\mathcal{O}_{y}\right)$ is connected. These facts imply that Supp $\Phi\left(\mathcal{O}_{y}\right)$ is contained in a single fiber of $\pi^{\prime}$.

Suppose to the contrary that $\operatorname{Supp} \Phi\left(\mathcal{O}_{y}\right) \cap \operatorname{Supp} \Phi\left(\mathcal{O}_{x}\right) \neq \emptyset$. Take a point $z$ in this nonempty set. Then, it follows from Lemma 2.3 that neither $\mathbb{R H o m}\left(\Phi\left(\mathcal{O}_{x}\right), \mathcal{O}_{z}\right)$ nor $\mathbb{R H o m}\left(\Phi\left(\mathcal{O}_{y}\right), \mathcal{O}_{z}\right)$ vanishes. Again Lemma 2.3 implies that $\operatorname{Supp} \Phi^{-1}\left(\mathcal{O}_{z}\right)$ contains the points $x$ and $y$. This induces

${ }^{2}$ If the fiber over the point $c$ is a multiple fiber of type ${ }_{m} \mathrm{I}_{n}$, then the reduced form is of type $\mathrm{I}_{n}$. 


\section{H. UEHARA}

a contradiction, because $\operatorname{Supp} \Phi^{-1}\left(\mathcal{O}_{z}\right)$ is contained in a single fiber of $\pi$ and $\pi(x) \neq \pi(y)$. Therefore, we obtain $\operatorname{Supp} \Phi\left(\mathcal{O}_{y}\right) \cap \operatorname{Supp} \Phi\left(\mathcal{O}_{x}\right)=\emptyset$.

For $y \in S$, denote the inclusion $S^{\prime} \cong y \times S^{\prime} \hookrightarrow S \times S^{\prime}$ by $i$. Notice that

$$
\left.\mathcal{P}\right|_{y \times S^{\prime}} \cong \Phi\left(\mathcal{O}_{y}\right) \quad \text { and }\left.\quad \operatorname{Supp} \mathcal{P}\right|_{y \times S^{\prime}}=i^{-1}(\operatorname{Supp} \mathcal{P})
$$

(see [Huy06, Lemma 3.29]). Claim 3.1 and (3.1) give

$$
\operatorname{dim} \operatorname{Supp} \mathcal{P}=2 \text { or } 3 \text {. }
$$

Claim 3.2. Let $V_{1}$ and $V_{1}^{\prime}$ be non-empty open subsets of $C$. Let us define $U_{1}:=\pi^{-1}\left(V_{1}\right)$ and $U_{1}^{\prime}:=\pi^{\prime-1}\left(V_{1}^{\prime}\right)$. Suppose that there is an isomorphism $\varphi_{U_{1}}: U_{1} \rightarrow U_{1}^{\prime}$. Then there exist an automorphism $\varphi_{C} \in$ Aut $C$ and an isomorphism $\varphi: S \rightarrow S^{\prime}$ extending $\varphi_{U_{1}}$ such that $\varphi_{C} \circ \pi=\pi^{\prime} \circ \varphi$ holds.

Proof. We may assume $V_{1}^{\prime} \neq C$ (otherwise the proof is done). The only proper connected subvarieties of $V_{1}^{\prime}$ are points. Hence $\left.\pi^{\prime}\right|_{U_{1}^{\prime}} \circ \varphi_{U_{1}}$ maps fibers of $\left.\pi\right|_{U_{1}}$ to points and, consequently, $\varphi_{U_{1}}$ maps fibers to fibers. Thus there is a bijection $\varphi_{V_{1}}: V_{1} \rightarrow V_{1}^{\prime}$ such that $\left.\varphi_{V_{1}} \circ \pi\right|_{U_{1}}=\left.\pi^{\prime}\right|_{U_{1}^{\prime}} \circ \varphi_{U_{1}}$. One can deduce that $\varphi_{V_{1}}$ is a morphism from the fact that the composition $\left.\varphi_{V_{1}} \circ \pi\right|_{U_{1}}$ is a morphism.

Since $C$ is a smooth projective curve, $\varphi_{V_{1}}$ extends to an isomorphism $\varphi_{C}$. Take the elimination of indeterminancies $S \leftarrow T \rightarrow S^{\prime}$ of $\varphi_{U_{1}}$. Then [BHPV04, Proposition III.8.4] assures that the morphism $T \rightarrow C$ uniquely factors through a relatively minimal elliptic fibration. Namely, $\varphi_{U_{1}}$ extends an isomorphism $\varphi$ in the statement.

Let us denote by $Z$ the union of all $(-2)$-curves on $S$. Note that the set $Z$ coincides with the union of all reducible fibers. We also denote by $U$ the complement of $Z$ in $S$, by $V$ the image of $U$ under $\pi$, and by $F$ a smooth fiber of $\pi$. We define $Z^{\prime}, U^{\prime}, V^{\prime}$ and $F^{\prime}$ on $S^{\prime}$ similarly.

Suppose that there is an isomorphism $\varphi: S \rightarrow S^{\prime}$. Then, since $S$ and $S^{\prime}$ have a unique elliptic fibration (see Section 2.5), it induces an automorphism $\varphi_{C}$ of $C$ which satisfies $\varphi_{C} \circ \pi=\pi^{\prime} \circ \varphi$. Moreover, an isomorphism $\varphi_{U}: U \rightarrow U^{\prime}$ extends to an isomorphism $\varphi: S \rightarrow S^{\prime}$ by Claim 3.2. In particular, we have

$$
\text { Aut } S \cong \text { Aut } U, \quad \operatorname{Aut}_{C} S \cong \operatorname{Aut}_{V} U
$$

\subsection{Autoequivalences associated with reducible fibers}

In this subsection, we show that every autoequivalence of $D(S)$ induces an autoequivalence of $D_{Z}(S)$.

LEMma 3.3. Take a point $x \in S$.

(i) There is at most one integer $i$ such that $H^{i}\left(\Phi\left(\mathcal{O}_{x}\right)\right) \neq 0$ but

$$
c_{1}\left(H^{i}\left(\Phi\left(\mathcal{O}_{x}\right)\right)\right) \cdot c_{1}\left(H^{i}\left(\Phi\left(\mathcal{O}_{x}\right)\right)\right)=0 .
$$

(ii) If $x \in Z$, then $\operatorname{Supp} \Phi\left(\mathcal{O}_{x}\right)$ is contained in $Z^{\prime}$.

Proof. (i) By equation (2.1), we have

$$
\chi\left(H^{i}\left(\Phi\left(\mathcal{O}_{x}\right)\right), H^{i}\left(\Phi\left(\mathcal{O}_{x}\right)\right)\right)=-c_{1}\left(H^{i}\left(\Phi\left(\mathcal{O}_{x}\right)\right)\right) \cdot c_{1}\left(H^{i}\left(\Phi\left(\mathcal{O}_{x}\right)\right)\right)=0,
$$

and hence

$$
\operatorname{dim} \operatorname{Ext}_{S^{\prime}}^{1}\left(H^{i}\left(\Phi\left(\mathcal{O}_{x}\right)\right), H^{i}\left(\Phi\left(\mathcal{O}_{x}\right)\right)\right) \geqslant 2 .
$$




\section{Autoequivalences of Elliptic surfaces}

But then, [BM01, Lemma 2.9] implies that there are no integers $i, j$ with $j \neq i$ such that

$$
\operatorname{dim} \operatorname{Ext}_{X}^{1}\left(H^{j}\left(\Phi\left(\mathcal{O}_{x}\right)\right), H^{j}\left(\Phi\left(\mathcal{O}_{x}\right)\right)\right) \neq 0 .
$$

(ii) We begin by noting that $\Phi\left(\mathcal{O}_{x}\right)$ is simple, because so is $\mathcal{O}_{x}$. First, let us consider the case $\operatorname{dim} \operatorname{Supp} \mathcal{P}=2$. Then, there is an irreducible component $W$ of $\operatorname{Supp} \mathcal{P}$ such that the restrictions $\left.p\right|_{W}: W \rightarrow S$ and $\left.p^{\prime}\right|_{W}: W \rightarrow S^{\prime}$ of the projections $p$ and $p^{\prime}$ are birational morphisms (see the proof of [Kaw02, Theorem 2.3]). We put

$$
q:=\left.\left.p^{\prime}\right|_{W} \circ p\right|_{W} ^{-1}: S \rightarrow S^{\prime} .
$$

But as Kawamata pointed out in [Kaw02, Lemma 4.2], the map $q$ is an isomorphism in codimension 1, and hence in the surface case, it is an isomorphism by [Har77, Chapter V, Lemma 5.1]. Therefore, if a $(-2)$-curve $G$ contains the point $x$, the $(-2)$-curve $q(G)$ contains the point $q(x) \in \operatorname{Supp} \Phi\left(\mathcal{O}_{x}\right)$. Because each $(-2)$-curve on $S^{\prime}$ and each $\operatorname{Supp} \Phi\left(\mathcal{O}_{x}\right)$ is always contained in a single fiber of $\pi^{\prime}$, the support $\operatorname{Supp} \Phi\left(\mathcal{O}_{x}\right)$ is contained in the set $Z^{\prime}$.

Next, let us consider the case $\operatorname{dim} \operatorname{Supp} \mathcal{P}=3$. Suppose that $\Phi\left(\mathcal{O}_{x}\right)$ is a sheaf on $S^{\prime}$, after replacing $\Phi$ with $\Phi \circ[n]$ for some $n \in \mathbb{Z}$. Take a point $y$ which is sufficiently near the point $x$, but not in $Z$. Then $\Phi\left(\mathcal{O}_{y}\right)$ is also a sheaf by Lemma 2.1, and both $\Phi\left(\mathcal{O}_{x}\right)$ and $\Phi\left(\mathcal{O}_{y}\right)$ are 1-dimensional by the assumption $\operatorname{dim} \operatorname{Supp} \mathcal{P}=3$. Claim 3.1 implies that we may assume that $\Phi\left(\mathcal{O}_{y}\right)$ is a sheaf on a smooth elliptic curve $F^{\prime}$. It follows from [IU05, Lemma 4.8] that $\Phi\left(\mathcal{O}_{y}\right)$ is a 1-dimensional simple $\mathcal{O}_{F^{\prime}}$-module. Hence, it is a stable locally free sheaf on $F^{\prime}$; see [Bri98, Remark 3.4]. Denote the Chern class of the stable sheaf $\Phi\left(\mathcal{O}_{y}\right)$ on the fiber $F^{\prime}$ by $\left(0, a F^{\prime},-b\right)$ for some integers $a$ and $b$. Then we know that $\left(a \lambda_{S^{\prime}}, b\right)=1$ (see the proof of [BM01, Proposition 4.4]), and hence there is the elliptic surface $J_{S^{\prime}}(a, b) \rightarrow C$ together with the universal sheaf $\mathcal{U}$ on $J_{S^{\prime}}(a, b) \times S^{\prime}$. For the point $w \in J_{S^{\prime}}(a, b)$ representing the stable sheaf $\Phi\left(\mathcal{O}_{y}\right)$, we have

$$
\mathcal{O}_{w} \cong\left(\Phi_{J_{S^{\prime}}(a, b) \rightarrow S^{\prime}}^{\mathcal{U}}\right)^{-1} \circ \Phi\left(\mathcal{O}_{y}\right)
$$

It follows that the kernel of the Fourier-Mukai transform $\left(\Phi_{J_{S^{\prime}}(a, b) \rightarrow S^{\prime}}^{\mathcal{U}}\right)^{-1} \circ \Phi$ has 2-dimensional support. Now, we can apply the case $\operatorname{dim} \operatorname{Supp} \mathcal{P}=2$ to see that $\operatorname{Supp}\left(\Phi_{J_{S^{\prime}}}^{\mathcal{U}}(a, b) \rightarrow S^{\prime}\right)^{-1} \circ \Phi\left(\mathcal{O}_{x}\right)$ is contained in the union of $(-2)$-curves on $J_{S^{\prime}}(a, b)$. Then, it follows from Remark 2.15 that $\operatorname{Supp} \Phi\left(\mathcal{O}_{x}\right)$ is contained in the set $Z^{\prime}$.

Finally, suppose $\operatorname{dim} \operatorname{Supp} \mathcal{P}=3$ and that $\Phi\left(\mathcal{O}_{x}\right)$ is not a shift of a sheaf. Take an integer $i$ such that $H^{i}\left(\Phi\left(\mathcal{O}_{x}\right)\right)$ is non-zero. Then the conclusion in statement (i) says that $c_{1}\left(H^{i}\left(\Phi\left(\mathcal{O}_{x}\right)\right)\right)$ is not a multiple of a fiber $F^{\prime}$. Because $H^{i}\left(\Phi\left(\mathcal{O}_{x}\right)\right)$ is contained in a single fiber, this means that Supp $H^{i}\left(\Phi\left(\mathcal{O}_{x}\right)\right)$ is contained in a reducible fiber, and hence in $Z^{\prime}$.

Note that if Supp $\Phi\left(\mathcal{O}_{x}\right)$ is contained in an irreducible fiber of $\pi^{\prime}$, every cohomology sheaf $H^{i}\left(\Phi\left(\mathcal{O}_{x}\right)\right)$ satisfies $c_{1}\left(H^{i}\left(\Phi\left(\mathcal{O}_{x}\right)\right)\right) \cdot c_{1}\left(H^{i}\left(\Phi\left(\mathcal{O}_{x}\right)\right)\right)=0$. Hence, Lemma 3.3(i) yields that $\Phi\left(\mathcal{O}_{x}\right)$ is a shift of a sheaf.

Applying Lemmas 2.4 and 3.3(ii), we obtain the following.

Proposition 3.4. There is a natural group homomorphism

$$
\iota_{Z}: \text { Auteq } D(S) \rightarrow \text { Auteq } D_{Z}(S) .
$$

Let us define

$$
\text { Auteq }^{\dagger} D_{Z}(S):=\operatorname{Im} \iota_{Z}
$$

The following is used in the proof of Lemma 3.7. 


\section{H. UEHARA}

Corollary 3.5. Take a point $x \in U$. Then we have $\operatorname{Supp} \Phi\left(\mathcal{O}_{x}\right) \subset U^{\prime}$.

Proof. We use Lemma 2.3. Take any point $y \in Z^{\prime}$. Then we have

$$
\mathbb{R} \operatorname{Hom}\left(\Phi\left(\mathcal{O}_{x}\right), \mathcal{O}_{y}\right) \cong \mathbb{R} \operatorname{Hom}\left(\mathcal{O}_{x}, \Phi^{-1}\left(\mathcal{O}_{y}\right)\right)=0
$$

by Lemma 3.3(ii). This implies the assertion.

\subsection{Autoequivalences associated with irreducible fibers: classification}

We begin Section 3.3 by classifying Fourier-Mukai transforms between elliptic surfaces without reducible fibers.

Proposition 3.6. Let $\pi: S \rightarrow C$ and $\pi^{\prime}: S^{\prime} \rightarrow C$ be elliptic surfaces without reducible fibers. Here we do not assume that $C$ is projective. Let $\Phi=\Phi_{S \rightarrow S^{\prime}}^{\mathcal{P}}$ be a Fourier-Mukai transform over $C$ such that $\operatorname{dim} \operatorname{Supp} \mathcal{P}=2$ or 3 .

(i) The object $\mathcal{P} \in D\left(S \times_{C} S^{\prime}\right)$ is a shift of a sheaf, flat over $S$ by the first projection.

(ii) The following are equivalent:

(1) $\operatorname{dim} \operatorname{Supp} \mathcal{P}=2$.

(2) There are points $x \in S$ and $y \in S^{\prime}$ such that $\Phi\left(\mathcal{O}_{x}\right) \cong \mathcal{O}_{y}$.

(3) There are a line bundle $\mathcal{L}$ on $S$ and an isomorphism $\varphi: S \rightarrow S^{\prime}$ over $C$ such that $\Phi \cong \varphi_{*}((-) \otimes \mathcal{L})$.

(iii) Suppose $\operatorname{dim} \operatorname{Supp} \mathcal{P}=3$. Then there are integers $a$ and $b$ with $\left(a \lambda_{S^{\prime}}, b\right)=1$, a universal sheaf $\mathcal{U}$ on $S^{\prime} \times J_{S^{\prime}}(a, b)$ and an isomorphism $\phi: J_{S^{\prime}}(a, b) \rightarrow S$ over $C$ such that

$$
\Phi \cong \Phi_{J_{S^{\prime}}(a, b) \rightarrow S^{\prime}}^{\mathcal{U}} \circ \phi^{*} .
$$

Proof. (i) Take any point $x \in S$. By Claim 3.1, the irreducibility of the fibers of $\pi^{\prime}$ and Lemma 3.3(i), the object $\Phi\left(\mathcal{O}_{x}\right)$ is a shift of a sheaf. Hence, [Bri99, Lemma 4.3] implies that $\mathcal{P}$ is a shift of a sheaf, flat over $S$ by the first projection.

(ii) As a consequence of assertion (i), the dimension of the support of $\left.\mathcal{P}\right|_{x \times S^{\prime}} \cong \Phi\left(\mathcal{O}_{x}\right)$ does not depend on the choice of a point $x \in S$. Hence, in statements (1) and (2), every $\Phi\left(\mathcal{O}_{x}\right)$ has finite support. Then we get statement (3) by Lemma 2.2. Here, recall that $\mathcal{P}$ is a sheaf on $S \times_{C} S^{\prime}$, and hence $\varphi$ is defined over $C$. Obviously, statement (3) implies statements (1) and (2).

(iii) The proof goes parallel to that of [BM01, Proposition 4.4] (see also the proof of Lemma 3.3(ii)). Let us take a general point $x \in S$, and denote the Chern class of the stable sheaf $\Phi\left(\mathcal{O}_{x}\right)$ on a smooth fiber $F^{\prime}$ by $\left(0, a F^{\prime},-b\right)$ for some integers $a$ and $b$. Then we know that $\left(a \lambda_{S^{\prime}}, b\right)=1$, and hence we can define an elliptic surface $J_{S^{\prime}}(a, b) \rightarrow C$ and a universal sheaf $\mathcal{U}$ on $S^{\prime} \times J_{S^{\prime}}(a, b)$. For the point $y \in J_{S^{\prime}}(a, b)$ representing a stable sheaf $\Phi\left(\mathcal{O}_{x}\right)$, we have

$$
\Phi^{-1} \circ \Phi_{J_{S^{\prime}}(a, b) \rightarrow S^{\prime}}^{\mathcal{U}}\left(\mathcal{O}_{y}\right) \cong \mathcal{O}_{x}
$$

Applying Lemma 2.2 and replacing a universal bundle $\mathcal{U}$ with $\mathcal{U} \otimes p_{J_{S^{\prime}}(a, b)}^{*} \mathcal{L}$ for a line bundle $\mathcal{L}$ on $J_{S^{\prime}}(a, b)$ if necessary, we obtain the assertion.

If $\pi$ has a reducible fiber, the implication from statement (2) to statement (3) in Proposition 3.6(ii) fails because of the existence of twist functors associated with $(-2)$-curves.

Now we can prove the following important observation.

Lemma 3.7. In the notation of Section 3.1, there is an automorphism $\delta(\Phi) \in$ Aut $C$ satisfying $\delta(\Phi)(V)=V^{\prime}$ such that $\left.\mathcal{P}\right|_{U \times U^{\prime}}$ is a shift of a coherent sheaf on $U_{\delta(\Phi)} \times_{V^{\prime}} U^{\prime}$. Here, $U_{\delta(\Phi)} \times_{V^{\prime}} U^{\prime}$ is the fiber product of $U$ and $U^{\prime}$ over $V^{\prime}$ via the morphisms $\left.(\delta(\Phi) \circ \pi)\right|_{U}$ and $\left.\pi^{\prime}\right|_{U^{\prime}}$. 


\section{Autoequivalences of Elliptic surfaces}

Proof. Below, we freely compose $\Phi=\Phi^{\mathcal{P}}$ with a shift functor if necessary. First, suppose $\operatorname{dim} \operatorname{Supp} \mathcal{P}=2$. Then for every $x \in U$, Corollary 3.5 implies that $\operatorname{Supp} \Phi\left(\mathcal{O}_{x}\right)$ is contained in an irreducible fiber, and then, we obtain from Lemma 3.3(i) and [Bri99, Lemma 4.3] that $\left.\mathcal{P}\right|_{U \times S^{\prime}}$ is a sheaf that is flat over $U$. In particular, the sheaf $\left.\Phi\left(\mathcal{O}_{x}\right) \cong \mathcal{P}\right|_{x \times U^{\prime}}$ has finite support. Thus, Lemma 2.2 implies that there is a morphism $\varphi_{U}: U \rightarrow U^{\prime}$ satisfying $\Phi\left(\mathcal{O}_{x}\right) \cong \mathcal{O}_{\varphi_{U}(x)}$.

Hence, we can apply Claim 3.2 to obtain the automorphism $\varphi_{C}$ of $C$. Note that $\left.\mathcal{P}\right|_{U \times U^{\prime}}$ is the structure sheaf of the graph of the morphism $\varphi_{U}$, and hence a sheaf on $U_{\varphi_{U}} \times_{V^{\prime}} U^{\prime}$. This $\varphi_{C}$ plays the role of $\delta(\Phi)$ in the assertion.

Next, we consider the case $\operatorname{dim} \operatorname{Supp} \mathcal{P}=3$. By the same argument as in the proof of Proposition 3.6, there are integers $a$ and $b$ with $\left(a \lambda_{S^{\prime}}, b\right)=1$ such that for every point $x \in U$, there is a point $y \in J_{U^{\prime}}(a, b)$ satisfying

$$
\Phi^{-1} \circ \Phi_{J_{S^{\prime}}(a, b) \rightarrow S^{\prime}}^{\mathcal{U}}\left(\mathcal{O}_{y}\right) \cong \mathcal{O}_{x}
$$

Note that we can regard $J_{U^{\prime}}(a, b)$ as the inverse image of $V^{\prime}$ by the elliptic fibration $J_{S^{\prime}}(a, b) \rightarrow C$. Let us denote by $\mathcal{Q} \in D\left(J_{S^{\prime}}(a, b) \times S\right)$ the kernel of $\Phi^{-1} \circ \Phi_{J_{S^{\prime}}}^{\mathcal{U}}(a, b) \rightarrow S^{\prime}$. Since $\operatorname{dim} \operatorname{Supp} \mathcal{Q}=2$, we can apply the above argument to $\mathcal{Q}$, and then we obtain the assertion for $\mathcal{Q}_{J_{U^{\prime}}(a, b) \times U}$. Since $\left.\mathcal{U}\right|_{J_{U^{\prime}}(a, b) \times U^{\prime}}$ is a coherent sheaf on $J_{U^{\prime}}(a, b) \times_{V^{\prime}} U^{\prime}$, we obtain the assertion for $\left.\mathcal{P}\right|_{U \times U^{\prime}}$.

Remark 3.8. For a point $x \in S$, we put $c:=\delta(\Phi)(\pi(x)) \in C$. Furthermore, if the point $x$ belongs to $U$, we know from the definition of $\delta$ that $\operatorname{Supp} \Phi\left(\mathcal{O}_{x}\right)$ is contained in the fiber $\pi^{\prime-1}(c)$. Recall that $\operatorname{Supp} \Phi\left(\mathcal{O}_{x}\right)=\operatorname{Supp} \mathcal{P} \cap\left(x \times S^{\prime}\right)$ by (3.1), and that $\operatorname{Supp} \Phi\left(\mathcal{O}_{x}\right)$ is contained in a single fiber. These facts imply that $\operatorname{Supp} \Phi\left(\mathcal{O}_{x}\right)$ is contained in $\pi^{\prime-1}(c)$ for any $x \in S$. Therefore, we conclude that $\mathcal{P}$ is an object of $D_{S_{\delta(\Phi)} \times_{C} S^{\prime}}\left(S \times S^{\prime}\right)$.

On the other hand, $\mathcal{P}$ is not necessarily an object of $D\left(S_{\delta(\Phi)} \times{ }_{C} S^{\prime}\right)$. For instance, consider a twist functor $T_{\mathcal{O}_{G}}$ for a (-2)-curve $G$ on $S$. Because the spherical object $T_{\mathcal{O}_{G}}\left(\mathcal{O}_{G}(2)\right)$ is simple, the computation in Example 2.8(i) and [Huy06, Corollary 3.15] imply that it is not of a form $k_{*} \alpha$ for an object $\alpha \in D\left(F_{c}\right)$, a fiber $F_{c}$ and the inclusion $k: F_{c} \hookrightarrow S$. Consequently, Lemma 2.5(i) tells us that the kernel of $T_{\mathcal{O}_{G}}$ is not an object of $D\left(S \times_{C} S\right)$. Here, note that $\delta\left(T_{\mathcal{O}_{G}}\right)=\mathrm{id}_{C}$.

Proposition 3.9. In the notation of Section 3.1, assume furthermore that $S=S^{\prime}$. Then, the Fourier-Mukai autoequivalence $\Phi=\Phi^{\mathcal{P}}$ of $D(S)$ induces a Fourier-Mukai autoequivalence $\iota_{U}(\Phi)$ of $D(U)$ over $V$, by restricting the kernel $\mathcal{P}$ to $U \times U$. This $\iota_{U}$ defines a group homomorphism

$$
\iota_{U}: \text { Auteq } D(S) \rightarrow \text { Auteq } D(U)
$$

satisfying

$$
\mathbb{L} i^{*} \circ \Phi \cong \iota_{U}(\Phi) \circ \mathbb{L} i^{*}
$$

where $i$ is the inclusion $U \hookrightarrow S$.

Proof. The statement is a direct consequence of Lemma 3.7 and the isomorphisms

$$
\operatorname{id}_{U} \cong \iota_{U}\left(\Phi \circ \Phi^{-1}\right) \cong \iota_{U}(\Phi) \circ \iota_{U}\left(\Phi^{-1}\right) .
$$

The isomorphism (3.2) follows from a direct computation.

Let us define

$$
\text { Auteq }^{\dagger} D(U):=\operatorname{Im} \iota_{U}
$$

Note that the elements of Auteq ${ }^{\dagger} D(U)$ are classified in Proposition 3.6. 


\section{H. UEHARA}

Remark 3.10. Lemma 3.7 tells us that there is a group homomorphism

$$
\delta: \text { Auteq } D(S) \rightarrow \text { Aut } C \text {. }
$$

The map $\delta$ factors through the map $\iota_{U}$, and hence it induces a map

$$
\delta_{U}: \operatorname{Auteq}^{\dagger} D(U) \rightarrow \text { Aut } V \quad(\cong \operatorname{Aut} C) .
$$

It then follows from Proposition 3.6 that

$$
\begin{aligned}
\operatorname{Im} \delta & =\left\{\varphi_{C} \mid \varphi \in \text { Aut } S \text { or } \varphi: S \rightarrow J_{S}(a, b) \text { isomorphism with }\left(a \lambda_{S}, b\right)=1\right\} \\
& =\left\{\varphi_{C} \mid \varphi \in \text { Aut } S \text { or } \varphi: S \rightarrow J_{S}(b) \text { isomorphism with }\left(\lambda_{S}, b\right)=1\right\} .
\end{aligned}
$$

The second equality follows from (2.5).

\subsection{Autoequivalences associated with irreducible fibers: structure of the group}

We use the notation of Section 3.1. The aim of Section 3.4 is to study the structure of the group Auteq ${ }^{\dagger} D(U)$.

For $m \in \mathbb{Z}$, we define the congruence subgroup of $\mathrm{SL}(2, \mathbb{Z})$ by

$$
\Gamma_{0}(m):=\left\{\left(\begin{array}{ll}
c & a \\
d & b
\end{array}\right) \in \mathrm{SL}(2, \mathbb{Z}) \mid d \in m \mathbb{Z}\right\} .
$$

Let us consider the surjective map

$$
\Gamma_{0}\left(\lambda_{S}\right) \rightarrow\left(\mathbb{Z} / \lambda_{S} \mathbb{Z}\right)^{*} / H_{S}, \quad\left(\begin{array}{cc}
c & a \\
d & b
\end{array}\right) \mapsto b .
$$

This is actually a group homomorphism and its kernel coincides with the group

$$
\left\{\left(\begin{array}{cc}
c & a \\
d & b
\end{array}\right) \in \Gamma_{0}\left(\lambda_{S}\right) \mid J_{S}(b) \cong S\right\}
$$

THEOREM 3.11. There is a short exact sequence

$$
\begin{aligned}
1 & \rightarrow\left\langle\otimes \mathcal{O}_{U}(D) \mid D \cdot F=0\right\rangle \rtimes \text { Aut } S \times \mathbb{Z}[2] \rightarrow \text { Auteq }^{\dagger} D(U) \rightarrow \\
& \rightarrow\left\{\left(\begin{array}{ll}
c & a \\
d & b
\end{array}\right) \in \Gamma_{0}\left(\lambda_{S}\right) \mid J_{S}(b) \cong S\right\} \rightarrow 1 .
\end{aligned}
$$

Proof. For $\Phi^{\mathcal{P}} \in$ Auteq $^{\dagger} D(U)$, note that $\mathcal{P}$ is a shift of a sheaf on $U_{\delta_{U}(\Phi)} \times_{V} U$, by Lemma 3.7.

Take a smooth fiber $F=F_{c}$ over a point $c \in V$, and denote by $F^{\prime}=F_{c^{\prime}}$ a smooth fiber over the point $c^{\prime}=\delta(\Phi)(c)$. Let $k: F \hookrightarrow U$ and $k^{\prime}: F^{\prime} \hookrightarrow U$ be the natural inclusions.

It follows from Lemma 2.5 that we obtain a Fourier-Mukai transform $\Phi_{F \rightarrow F^{\prime}}^{\left.\mathcal{P}\right|_{F \times F^{\prime}}}$ such that there is an isomorphism of functors

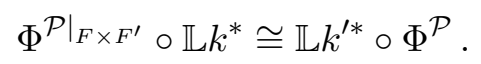

If we choose a basis as in Section 2.4, there is an identification between $H^{\mathrm{ev}}(F, \mathbb{Z})$ and $H^{\mathrm{ev}}\left(F^{\prime}, \mathbb{Z}\right)$. We then obtain a group homomorphism

$$
\Theta_{U}: \text { Auteq }^{\dagger} D(U) \rightarrow \mathrm{SL}(2, \mathbb{Z}) .
$$

Notice that this morphism does not depend on the choice of a smooth fiber $F$, by the classification of the elements of Auteq ${ }^{\dagger} D(U)$ in Proposition 3.6. 


\section{Autoequivalences of Elliptic surfaces}

Since $F$ and $F^{\prime}$ are isomorphic, we fix an isomorphism. Then $\Phi_{F \rightarrow F^{\prime}}^{\left.\mathcal{P}\right|_{F \times F^{\prime}}}$ can be regarded as an autoequivalence of $D(F)$, and we have

$$
\Theta_{U}\left(\Phi^{\mathcal{P}}\right)=\theta\left(\Phi_{F \rightarrow F^{\prime}}^{\left.\mathcal{P}\right|_{F \times F^{\prime}}}\right)
$$

This is important for a later computation. (Recall the definition of $\theta$ in Section 2.4.)

Suppose $\Phi^{\mathcal{P}} \in \operatorname{Ker} \Theta_{U}$. Proposition 3.6 implies $\operatorname{dim} \operatorname{Supp} \mathcal{P}=2$, and hence

$$
\Phi^{\mathcal{P}} \in\left\langle\otimes \mathcal{O}_{U}(D) \mid D \cdot F=0\right\rangle \rtimes \text { Aut } S \times \mathbb{Z}[2] ;
$$

that is,

$$
\operatorname{Ker} \Theta_{U} \cong\left\langle\otimes \mathcal{O}_{U}(D) \mid D \cdot F=0\right\rangle \rtimes \text { Aut } S \times \mathbb{Z}[2] .
$$

Next, let us consider the image of $\Theta_{U}$. Take integers $a$ and $b$ with $a>0$ and $\left(a \lambda_{S}, b\right)=1$. Assume that there is an isomorphism $\phi: J_{S}(a, b) \rightarrow S$. Then

$$
\Phi_{J_{S}(a, b) \rightarrow S}^{\mathcal{U}} \circ \phi^{*}
$$

gives an autoequivalence of $D(S)$. In this case, it follows from Remark 2.11 that

$$
\Theta_{U} \circ \iota_{U}\left(\Phi_{J_{S}(a, b) \rightarrow S}^{\mathcal{U}} \circ \phi^{*}\right)=\left(\begin{array}{ll}
c & a \\
d & b
\end{array}\right) \in \mathrm{SL}(2, \mathbb{Z})
$$

holds for some $c, d \in \mathbb{Z}$ such that $\lambda_{S}$ divides $d$. We also have

$$
\Theta_{U} \circ \iota_{U}(\operatorname{Pic} S)=\left\langle\left(\begin{array}{cc}
1 & 0 \\
\lambda_{S} & 1
\end{array}\right)\right\rangle
$$

and

$$
\Theta_{U}([1])=\left(\begin{array}{cc}
-1 & 0 \\
0 & -1
\end{array}\right)
$$

Then it follows from Proposition 3.6 that

$$
\operatorname{Im} \Theta_{U}=\left\langle\left(\begin{array}{cc}
1 & 0 \\
\lambda_{S} & 1
\end{array}\right),\left(\begin{array}{cc}
c & a \\
d & b
\end{array}\right),\left(\begin{array}{cc}
-1 & 0 \\
0 & -1
\end{array}\right) \mid a \neq 0, b c-a d=1, d \in \lambda_{S} \mathbb{Z}, J_{S}(b) \cong S\right\rangle .
$$

Note that

$$
\left(\begin{array}{cc}
-1 & 0 \\
\lambda_{S} & -1
\end{array}\right)=\left(\begin{array}{cc}
1 & 0 \\
-\lambda_{S} & 1
\end{array}\right)\left(\begin{array}{cc}
-1 & 0 \\
0 & -1
\end{array}\right) \in \operatorname{Im} \Theta_{U}
$$

Furthermore, we can see

$$
\left(\begin{array}{cc}
-1 & 0 \\
0 & -1
\end{array}\right)=\left(\begin{array}{cc}
-1 & a \\
0 & -1
\end{array}\right)\left(\begin{array}{ll}
1 & a \\
0 & 1
\end{array}\right)
$$

for any $a \in \mathbb{Z}$. Therefore, we conclude that

$$
\operatorname{Im} \Theta_{U}=\left\{\left(\begin{array}{cc}
c & a \\
d & b
\end{array}\right) \in \Gamma_{0}\left(\lambda_{S}\right) \mid J_{S}(b) \cong S\right\},
$$

which completes the proof of Theorem 3.11.

We define

$$
\Theta: \text { Auteq } D(S) \rightarrow \mathrm{SL}(2, \mathbb{Z})
$$

to be the composition $\Theta_{U} \iota_{U}$, where we regard $\iota_{U}$ as a surjective homomorphism from Auteq $D(S)$ to Auteq ${ }^{\dagger} D(U)$. Thus, by definition, $\operatorname{Im} \Theta=\operatorname{Im} \Theta_{U}$. 


\section{H. UEHARA}

Remark 3.12. (i) By the remark before Theorem 3.11, we have a group isomorphism

$$
\Gamma_{0}\left(\lambda_{S}\right) / \operatorname{Im} \Theta \cong\left(\mathbb{Z} / \lambda_{S} \mathbb{Z}\right)^{*} / H_{S} .
$$

As explained in Section 2.6, the latter group can be naturally identified with the set $\operatorname{FM}(S)$.

(ii) When $\left|\left(\mathbb{Z} / \lambda_{S} \mathbb{Z}\right)^{*}\right| \leqslant 2$ (for example, $\left.\lambda_{S} \leqslant 4\right)$, we have $\left(\mathbb{Z} / \lambda_{S} \mathbb{Z}\right)^{*}=H_{S}$. Hence, we can see that $\operatorname{Im} \Theta=\Gamma_{0}\left(\lambda_{S}\right)$. In particular, when $\lambda_{S}=1$, we see that $\operatorname{Im} \Theta=\operatorname{SL}(2, \mathbb{Z})$.

\subsection{The kernels of $\iota_{Z}$ and $\iota_{U}$}

Now, let us study the kernel of the homomorphisms $\iota_{Z}$ given in Proposition 3.4. First of all, we may assume $Z \neq \emptyset$ because, otherwise, we have

$$
\operatorname{Ker} \iota_{Z}=\text { Auteq } D(S)=\text { Auteq }^{\dagger} D(U),
$$

and the last group was already studied in Section 3.4. Take $\Phi \in \operatorname{Ker} \iota_{Z}$. Then for any $x \in Z$, we have $\Phi\left(\mathcal{O}_{x}\right) \cong \mathcal{O}_{x}$. Hence, by [Huy06, Corollary 6.14], there is a point $y \in U$ such that

$$
\Phi\left(\mathcal{O}_{y}\right) \cong \mathcal{O}_{w}
$$

for some $w \in U$. We can apply Proposition 3.6(ii) for $\iota_{U}(\Phi)$ to obtain that for all points $y \in U$, there is a point $w$ satisfying (3.4). Therefore, Lemma 2.2 implies that Ker $\iota_{Z}$ is contained in the group $A(S)$ of the standard autoequivalences (see Section 1.1), and hence

$$
\text { Ker } \left.\iota_{Z}=\left\langle\otimes \mathcal{O}_{S}\left(F_{c}\right)\right| F_{c} \text { an irreducible fiber of } \pi\right\rangle \rtimes \operatorname{Aut}_{Z} S,
$$

where $\operatorname{Aut}_{Z} S:=\{\varphi \in$ Aut $S \mid \varphi(z)=z$ for all $z \in Z\}$.

Let us set

$$
\left.B:=\left\langle T_{\mathcal{O}_{G}(a)}\right| G \text { is a }(-2) \text {-curve }\right\rangle .
$$

For the homomorphism $\iota_{U}$ given in Proposition 3.9, we believe that the equality

$$
B=\operatorname{Ker} \iota_{U}
$$

holds. Actually, we have the following.

Lemma 3.13. Suppose that equality (3.6) holds. Then Conjecture 1.1 is true.

Proof. The result follows from the description of Auteq ${ }^{\dagger} D(U)$ in Theorem 3.11.

In Sections 4, 5 and 6, we shall check equality (3.6) in the case that all reducible fibers on a given projective elliptic surface are of type $\mathrm{I}_{n}(n>1)$, and consequently show Conjecture 1.1 in this case.

\section{Autoequivalences associated with singular fibers of type $I_{n}$ for $n>1$}

Throughout this section, $\pi: S \rightarrow C$ is a projective elliptic surface whose reducible fibers are nonmultiple cycles of $(-2)$-curves, that is, of type $\mathrm{I}_{n}$ for $n>1$. In this case, the set $Z$ is a disjoint union of cycles of projective lines. Below, we regard $Z$ as a closed subscheme of $S$ equipped with the reduced induced structure.

In our setting, line bundles on (-2)-curves are spherical in the sense of Definition-Proposition 2.6. Therefore, Auteq $D_{Z}(S)$ contains twist functors, and hence it is highly involved. The following is the main result of this article. 


\section{Autoequivalences of Elliptic surfaces}

TheOrEm 4.1. Let $S$ be a smooth projective elliptic surface with $\kappa(S) \neq 0$. Suppose that each reducible fiber on the elliptic surface $S$ is of type $\mathrm{I}_{n}$ for some $n>1$. Then Conjecture 1.1 is true. Namely, we have the exact sequence

$$
\begin{aligned}
1 \rightarrow\left\langle B, \otimes \mathcal{O}_{S}(D) \mid D \cdot F=0\right\rangle & \rtimes \text { Aut } S \times \mathbb{Z}[2] \rightarrow \text { Auteq } D(S) \\
& \stackrel{\Theta}{\rightarrow}\left\{\left(\begin{array}{ll}
c & a \\
d & b
\end{array}\right) \in \Gamma_{0}\left(\lambda_{S}\right) \mid J_{S}(a, b) \cong S\right\} \rightarrow 1 .
\end{aligned}
$$

Theorem 4.1 concerns the autoequivalences of the derived categories of surfaces containing $\tilde{A}_{n}$-configurations of (-2)-curves. Many ideas of the proof come from [IU05], where we study the autoequivalences of the derived categories of surfaces containing $A_{n}$-configurations of $(-2)$ curves.

\subsection{Reduction of the proof of Theorem 4.1}

Recall that Lemma 3.13 tells us that if we can show equation (3.6), we obtain Theorem 4.1.

Let $\left\{Z_{j}\right\}_{j=1}^{M}$ be the set of connected components of $Z$; that is, each $Z_{j}$ is a singular fiber of type $\mathrm{I}_{n_{j}}$ for some $n_{j}>1$. We define

$$
\left.B_{j}:=\left\langle T_{\mathcal{O}_{G}(a)}\right| G \text { is a }(-2) \text {-curves contained in } Z_{j}\right\rangle .
$$

Take a connected component of $Z$, and denote it by $Z_{0}$. We put

$$
Z_{0}=C_{1} \cup \cdots \cup C_{n}
$$

such that each $C_{i}$ is a $(-2)$-curve on $S$ and the $C_{i}$ satisfy

$$
C_{l} \cdot C_{m}= \begin{cases}1, & |l-m|=1 \text { or }|l-m|=n-1, \\ 0, & \text { otherwise }\end{cases}
$$

in the case $n>2$. In the case $n=2$, the curves $C_{1}$ and $C_{2}$ intersect each other transversely at two points.

The inclusion $B \subset \operatorname{Ker} \iota_{U}$ holds by Remark 2.7. Thus, in order to prove (3.6), it is left to show Ker $\iota_{U} \subset B$. This, in turn, can be reduced to showing the following.

Proposition 4.2 (cf. [IU05, Proposition 1.7]). Suppose that we are given an autoequivalence $\Phi$ of $D_{Z_{0}}(S)$ preserving the cohomology class $\operatorname{ch}\left(\mathcal{O}_{x}\right) \in H^{4}(S, \mathbb{Q})$ for all points $x \in Z_{0}$. Then, there are integers $a, b(1 \leqslant b \leqslant n)$ and $i$ and an autoequivalence $\Psi \in B_{0}$ such that

$$
\Psi \circ \Phi\left(\mathcal{O}_{C_{1}}\right) \cong \mathcal{O}_{C_{b}}(a)[i] \text { and } \Psi \circ \Phi\left(\mathcal{O}_{C_{1}}(-1)\right) \cong \mathcal{O}_{C_{b}}(a-1)[i] .
$$

Consequently, for any point $x \in C_{1}$, we can find a point $y \in C_{b}$ with $\Psi \circ \Phi\left(\mathcal{O}_{x}\right) \cong \mathcal{O}_{y}[i]$.

Suppose that we have shown Proposition 4.2. Note that $\operatorname{Ker} \iota_{U} \cap \operatorname{Ker} \iota_{Z}=\{1\}$ by (3.5). Thus we can consider both $\operatorname{Ker} \iota_{U}$ and $B$ as subgroups of Auteq $D_{Z}(S)$ and prove the inclusion Ker $\iota_{U} \subset B$ inside this group.

Take $\Phi \in \operatorname{Ker} \iota_{U}$. Since $\delta(\Phi)=\operatorname{id}_{C}$ for $\delta$ as constructed in Section 3.3, we see that $\Phi$ induces autoequivalences $\Phi_{j}$ of $D_{Z_{j}}(S)$. Since all points $x \in S$ define the same cohomology class $\operatorname{ch}\left(\mathcal{O}_{x}\right)$, the autoequivalence $\Phi_{j}$ satisfies the assumption in Proposition 4.2. We fix some $j$ and put $n=n_{j}$ for simplicity. We take the irreducible decomposition of $Z_{j}$ as $Z_{j}=C_{1} \cup \cdots \cup C_{n}$. Now, we can apply Proposition 4.2 for $\Phi_{j}$ to find $\Psi_{j} \in B_{j}$ satisfying (4.2). Since $\Psi_{j} \circ \Phi$ also belongs to $\operatorname{Ker} \iota_{U}$, we have $b=1$ and $i=0$, and $x=y$ in Proposition 4.2 . Hence, $\Psi_{j} \circ \Phi_{j}$ gives an autoequivalence 


\section{H. UEHARA}

of $D_{Z_{j}^{\prime}}(S)$, where $Z_{j}^{\prime}=C_{2} \cup \cdots \cup C_{n}$. Since $Z_{j}^{\prime}$ is an $A_{n-1}$-configuration of (-2)-curves, [IU05, Theorem 1.3] implies that

$$
\begin{aligned}
\Psi_{j} \circ \Phi_{j} \in & \left(\left(\left\langle B_{j}^{\prime}, \operatorname{Pic} S\right\rangle \rtimes \text { Aut } S\right) \times \mathbb{Z}\right) \cap \operatorname{Ker} \iota_{U} \\
& \cong B_{j}^{\prime} \rtimes\left\langle\otimes \mathcal{O}_{S}\left(C_{i}\right) \mid i=1, \ldots, n\right\rangle \\
& \subset B_{j},
\end{aligned}
$$

where we put $B_{j}^{\prime}:=\left\langle T_{\mathcal{O}_{C_{i}}(a)} \mid i=2, \ldots, n\right\rangle$ and the last inclusion is a consequence of [IU05, Proposition 4.18(i)]. Hence, we know that $\Phi_{j} \in B_{j}$.

We apply this argument for each $Z_{j}(1 \leqslant j \leqslant M)$, and then we can see that

$$
\Psi_{1} \circ \cdots \circ \Psi_{M} \circ \Phi \in B
$$

and hence $\Phi \in B$. Therefore, we have $\operatorname{Ker} \iota_{U} \subset B$, as desired.

Remark 4.3. In the $A_{n}$-case of Proposition 4.2, that is, [IU05, Proposition 1.7], we do not need the assumption that $\Phi$ preserves the cohomology class $\operatorname{ch}\left(\mathcal{O}_{x}\right)$, since it is always true (up to the

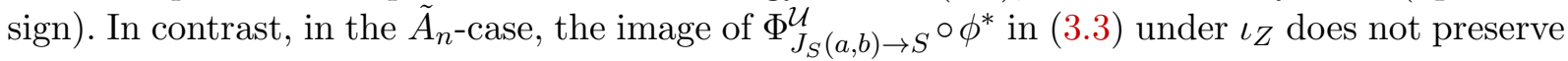
the cohomology class $\operatorname{ch}\left(\mathcal{O}_{x}\right)$. The existence of such elements forces us to put this assumption in Proposition 4.2.

The subgroup

$$
\left\langle B,\left(\operatorname{Pic} S /\left\langle\otimes \mathcal{O}_{S}\left(F_{c}\right) \mid c \in V\right\rangle\right) \rtimes\left(\operatorname{Aut} S / \operatorname{Aut}_{Z} S\right) \times \mathbb{Z}[1]\right\rangle
$$

of Auteq ${ }^{\dagger} D_{Z}(S)$ preserves $H^{4}(S, \mathbb{Q})$, and therefore it is strictly smaller than $\operatorname{Auteq}^{\dagger} D_{Z}(S)$. This is in contrast to the $A_{n}$-case of Theorem 2.9 .

We shall prove Proposition 4.2 in Section 6. As an intermediate step, we first show the following.

Proposition 4.4 (cf. [IU05, Proposition 1.6]). Let $\alpha$ be a spherical object in $D_{Z_{0}}(S)$. Then there are integers $a, b(1 \leqslant b \leqslant n)$ and $i$ and an autoequivalence $\Psi \in B_{0}$ such that

$$
\Psi(\alpha) \cong \mathcal{O}_{C_{b}}(a)[i] .
$$

Proposition 4.4 is proved in Section 5.

\subsection{Cohomology sheaves of spherical objects}

4.2.1 Torsion-free sheaves on a chain of projective lines. We consider the cycle of lines $Z_{0}=C_{1} \cup \cdots \cup C_{n}$ as an abstract variety and denote it by $\tilde{\mathbb{I}}_{n}$. The curves $C_{i}$ are labelled as in (4.1) when $n>2$.

We denote by $\mathbb{I}_{n}$ a chain of $n$ projective lines. We put $\mathbb{I}_{n}=C_{1}^{\prime} \cup \cdots \cup C_{n}^{\prime}$, where each $C_{i}^{\prime}$ is a projective line and the $C_{i}^{\prime}$ satisfy

$$
C_{l}^{\prime} \cdot C_{m}^{\prime}= \begin{cases}1, & |l-m|=1, \\ 0, & \text { otherwise }\end{cases}
$$

For a coherent sheaf $\mathcal{R}$ on $\mathbb{I}_{n}$ or $\tilde{\mathbb{I}}_{n}$, we denote by $\operatorname{deg}_{C} \mathcal{R}$ the degree of the restriction $\left.\mathcal{R}\right|_{C}$ to the component $C$ of $\mathbb{I}_{n}$ or $\tilde{\mathbb{I}}_{n}$. It is known that a line bundle $\mathcal{L}$ on $\mathbb{I}_{n}$ is determined by the degree $\left.\mathcal{L}\right|_{C}$ on all the components $C$; that is,

$$
\operatorname{Pic} \mathbb{I}_{n} \cong \mathbb{Z}^{n} \text {. }
$$




\section{Autoequivalences of Elliptic surfaces}

The line bundle corresponding to the vector $\left(a_{1}, \ldots, a_{n}\right) \in \mathbb{Z}^{n}$ is denoted by

$$
\mathcal{O}_{\mathbb{I}_{n}}\left(a_{1}, \ldots, a_{n}\right) .
$$

When we write $*$ instead of $a_{l}$, we do not specify the degree at $C_{l}^{\prime}$. For instance, when we write

$$
\mathcal{R}_{1}=\mathcal{O}_{\mathbb{I}_{3}}(a, b, *),
$$

this means that $\mathcal{R}_{1}$ is a line bundle on $\mathbb{I}_{3}$ such that $\operatorname{deg}_{C_{1}^{\prime}} \mathcal{R}_{1}=a, \operatorname{deg}_{C_{2}^{\prime}} \mathcal{R}_{1}=b$ and $\operatorname{deg}_{C_{3}^{\prime}} \mathcal{R}_{1}$ is arbitrary. The expression

$$
\mathcal{R}_{2}=\mathcal{O}_{C_{1}^{\prime} \cup \ldots}(a, *)
$$

means that $\mathcal{R}_{2}=\mathcal{O}_{\mathbb{I}_{k}}(a, *, \ldots, *)$ for some (not further specified) $k \geqslant 2$. Note that the support of $\mathcal{R}_{2}$ is strictly larger than $C_{1}^{\prime}$. We often use figures such as

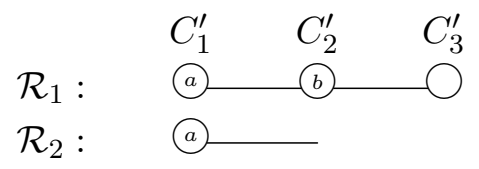

to define $\mathcal{R}_{1}$ and $\mathcal{R}_{2}$ above. We use a dashed line

$$
\begin{array}{ll} 
& C_{1}^{\prime} \\
\mathcal{R}_{3}: & \left(a-{ }_{-}\right.
\end{array}
$$

to indicate that $\mathcal{R}_{3}$ is either $\mathcal{O}_{C_{1}^{\prime}}(a)$ or $\mathcal{O}_{C_{1}^{\prime} \cup \ldots}(a, *)$.

4.2.2 Torsion-free, but not locally free sheaves on $\tilde{\mathbb{I}}_{n}$. For $m \in \mathbb{Z}$, we set

$$
C_{m}:=C_{i},
$$

where $i$ is the unique number with $1 \leqslant i \leqslant n$ and $m-i \in n \mathbb{Z}$.

Let $\mathcal{S}$ be an indecomposable sheaf on $\tilde{\mathbb{I}}_{n}$, pure of dimension 1 , but whose support is not the whole circle $\tilde{\mathbb{I}}_{n}$. Since $\operatorname{Supp} \mathcal{S}$ is connected, it must be of the form $C_{s} \cup C_{s+1} \cup \cdots \cup C_{s+k-1} \cong \mathbb{I}_{k}$ for some $s \in \mathbb{Z}$ and $1 \leqslant k \leqslant n-1$. Let $p_{s, k}: \mathbb{I}_{k} \rightarrow \tilde{\mathbb{I}}_{n}$ denote the embedding of $C_{s} \cup \cdots \cup C_{s+k-1}$. Then, by the results explained in the previous subsection,

$$
\mathcal{S} \cong \mathcal{S}_{s}\left(a_{s}, \ldots, a_{s+k-1}\right):=p_{s, k *} \mathcal{O}_{\mathbb{I}_{k}}\left(a_{s}, \ldots, a_{s+k-1}\right)
$$

for some integers $a_{s}, \ldots, a_{s+k-1}$.

For $k \geqslant n$, we fix a finite surjective morphism $p_{s, k}: \mathbb{I}_{k} \rightarrow \tilde{\mathbb{I}}_{n}$ such that for, $l=1, \ldots, k$, the restriction $\left.p_{s, k}\right|_{C_{l}^{\prime}}$ is an isomorphism with the image $C_{s+l-1}$ (one can think of $p_{s, k}$ as realizing $\mathbb{I}_{k}$ as a helix over the circle $\left.\tilde{\mathbb{I}}_{n}\right)$. Then, for $a_{s}, \ldots, a_{s+k-1} \in \mathbb{Z}$ we again set

$$
\mathcal{S}_{s}\left(a_{s}, \ldots, a_{s+k-1}\right):=p_{s, k *} \mathcal{O}_{\mathbb{I}_{k}}\left(a_{s}, \ldots, a_{s+k-1}\right) .
$$

For any $k \geqslant 1$, we also denote $\mathcal{S}_{s}\left(a_{s}, \ldots, a_{s+k-1}\right)$ by

$$
\mathcal{S}_{C_{s} \cup \ldots \cup C_{s+k-1}}\left(a_{s}, \ldots, a_{s+k-1}\right) .
$$

Notice that for $k<n$, we have

$$
\mathcal{S}_{C_{1} \cup \cdots \cup C_{k}}(0, \ldots, 0)=\mathcal{S}_{1}(\overbrace{0, \ldots, 0}^{k}) \cong \mathcal{O}_{C_{1} \cup \cdots \cup C_{k}},
$$

but in contrast

$$
\mathcal{S}_{C_{1} \cup \cdots \cup C_{n}}(0, \ldots, 0)=\mathcal{S}_{1}(\overbrace{0, \ldots, 0}^{n}) \nRightarrow \mathcal{O}_{\tilde{\mathbb{I}}_{n}} .
$$




\section{H. UEHARA}

We can see that

$$
\left.\mathcal{S}_{s}\left(a_{s}, \ldots, a_{s+k-1}\right)\right|_{C_{m}} \cong \bigoplus_{l \in m+n \mathbb{Z}, s \leqslant l \leqslant s+k-1} \mathcal{O}_{C_{m}}\left(a_{l}\right)
$$

Proposition 4.5 ([BBDG06, Theorem 19]). Every indecomposable sheaf $\mathcal{S}$ on $\tilde{\mathbb{I}}_{n}$ which is torsion free but not locally free is of the form $\mathcal{S} \cong \mathcal{S}_{s}\left(a_{s}, \ldots, a_{s+k-1}\right)$ for some $k>0$ and $a_{s}, \ldots, a_{s+k-1} \in \mathbb{Z}$.

4.2.3 Cohomology sheaves of spherical objects. Henceforth, we freely use the notation and results on $\tilde{\mathbb{I}}_{n}$ mentioned above.

For a complex analytic open subset $U$ of $S$ and a spherical object $\alpha \in D(S)$, let

$$
\Sigma(\alpha)_{U}
$$

be the set of all indecomposable summands of the coherent $\mathcal{O}_{U}$-module $\left.\bigoplus_{p} H^{p}(\alpha)\right|_{U}$. If $U=S$, we just denote it by

$$
\Sigma(\alpha)
$$

We frequently use the following.

Lemma 4.6. (i) For a spherical object $\alpha \in D_{Z_{0}}(S)$, the direct sum $\bigoplus_{p} H^{p}(\alpha)$ of its cohomology sheaves is rigid as an $\mathcal{O}_{S}$-module and torsion free as an $\mathcal{O}_{Z_{0}}$-module.

(ii) Any $\mathcal{R} \in \Sigma(\alpha)$ cannot be a locally free $\mathcal{O}_{Z_{0}}$-module, and is of the form $\mathcal{S}_{s}\left(a_{s}, a_{s+1}, \ldots, a_{t}\right)$ for some integers $s$ and $t$ with $C_{s-1} \neq C_{t}$.

Proof. (i) This is a direct consequence of [IU05, Proposition 4.5, Lemmas 4.8 and 4.9].

(ii) For a torsion-free sheaf $\mathcal{E}$ on $Z_{0}$ such that $c_{1}(\mathcal{E})$ is some multiple of $\left[Z_{0}\right]$, we can see that $\chi_{S}(\mathcal{E}, \mathcal{E})=-c_{1}(\mathcal{E})^{2}=0$, which implies that $\mathcal{E}$ is not rigid. Hence, the torsion freeness and the rigidity of $\mathcal{R}$ by assertion (i) imply the result.

Remark 4.7. Suppose that $S$ has a multiple fiber $m Z_{0}$, that is, a fiber of type ${ }_{m} \mathrm{I}_{n}$ for some $m>1$, $n>0$. Then [IU05, Lemma 4.8] implies that $\bigoplus_{p} H^{p}(\alpha)$ is an $\mathcal{O}_{m Z_{0}}$-module. In particular, we cannot conclude that Lemma 4.6(i) holds. This is the reason why we assume that each reducible fiber is non-multiple in Theorem 4.1.

For a connected union of $(-2)$-curves

$$
Z^{\prime}:=C_{s} \cup C_{s+1} \cup \cdots \cup C_{t}\left(\varsubsetneqq Z_{0}\right),
$$

take a sufficiently small complex analytic neighbourhood of $Z^{\prime}$, and denote it by

$$
U_{s, \ldots, t} \text {. }
$$

Here, sufficiently small means that

$$
Z_{0} \cap U_{s, \ldots, t} \cong D_{s-1} \cup C_{s} \cup \cdots \cup C_{t} \cup D_{t+1}
$$

where $D_{i}$ is a 1-dimensional complex disk.

For a given $\beta \in D_{Z_{0}}(S)$, define

$$
l_{i}(\beta):=\sum_{p} \operatorname{length}_{\mathcal{O}_{S, \eta_{i}}} H^{p}(\beta)_{\eta_{i}}
$$




\section{Autoequivalences of Elliptic surfaces}

for each curve $C_{i}$, where $\mathcal{O}_{S, \eta_{i}}$ is the local ring of $S$ at the generic point $\eta_{i}$ of $C_{i}$, the object $H^{p}(\beta)_{\eta_{i}}$ is the stalk over $\eta_{i}$ and length $\mathcal{O}_{S, \eta_{i}}$ measures the length over $\mathcal{O}_{S, \eta_{i}}$. Furthermore, define

$$
l(\beta):=\sum_{i=1}^{n} l_{i}(\beta),
$$

and also define

$$
l\left(\left.\beta\right|_{U}\right):=\sum_{i: U \cap C_{i} \neq \emptyset} l_{i}(\beta)
$$

for a complex analytic open subset $U$ of $S$. These invariants play important roles in the induction step in the proofs of Propositions 4.2 and 4.4.

Using Lemma 4.6, we can deduce strong conditions on the elements of $\Sigma(\alpha)$ such as Lemma 4.9 below. Before giving a general statement in Lemma 4.9, we first give a special one for the case $n=2$, since the proof is slightly different from the one in the case $n>2$.

LEMma 4.8. Let us consider the case $n=2$, that is, $Z_{0} \cong \tilde{\mathbb{I}}_{2}$, and let $\alpha$ be a spherical object in $D_{Z_{0}}(S)$. Assume that there is an element

$$
\mathcal{S}:=\mathcal{S}_{s}\left(a_{s}, a_{s+1}, \ldots, a_{t}\right) \in \Sigma(\alpha)
$$

with $s \neq t$, which means that $l(\mathcal{S})>1$. Then there are integers $a$ and $i$ with $i=1$ or 2 satisfying the following conditions:

(i) We have $C_{i}=C_{s}=C_{t}$ and $a=a_{s}=a_{t}$. The integers $i$ and $a$ are independent of the choice of $\mathcal{S} \in \Sigma(\alpha)$ with $l(\mathcal{S})>1$.

(ii) Assume that there is an element $\mathcal{S}^{\prime} \in \Sigma(\alpha)$ with $l\left(\mathcal{S}^{\prime}\right)=1$. Then $\mathcal{S}^{\prime}=\mathcal{O}_{C_{i}}(b)$ with $b=a$ or $a-1$.

(iii) In the situation of condition (ii), assume furthermore that the above $\mathcal{S}$ satisfies $l(\mathcal{S})>3$. Then we have

$$
a_{s+2}=a_{s+4}=\cdots=a_{t-2}=b+1 .
$$

Proof. Take elements

$$
\mathcal{S}_{1}:=\mathcal{S}_{s_{1}}\left(a_{s_{1}}, \ldots, a_{t_{1}}\right), \quad \mathcal{S}_{2}:=\mathcal{S}_{s_{2}}\left(b_{s_{2}}, \ldots, b_{t_{2}}\right) \in \Sigma(\alpha) .
$$

First, we show $C_{s_{1}}=C_{t_{2}}$. Suppose to the contrary that $C_{s_{1}} \neq C_{t_{2}}$, namely $C_{s_{1}}=C_{t_{2}+1}$ holds. Then there is a non-split exact sequence

$$
0 \rightarrow \mathcal{S}_{2} \rightarrow \mathcal{S}_{s_{2}}\left(b_{s_{2}}, \ldots, b_{t_{2}-1}, b_{t_{2}}+1, a_{s_{1}}, \ldots, a_{t_{1}}\right) \rightarrow \mathcal{S}_{1} \rightarrow 0,
$$

which gives a contradiction with the rigidity of $\mathcal{S}_{1} \oplus \mathcal{S}_{2}$ in Lemma 4.6(i). Thus, we obtain $C_{s_{1}}=C_{t_{2}}$.

If we interchange the roles of $\mathcal{S}_{1}$ and $\mathcal{S}_{2}$ in the above argument, then we obtain $C_{s_{2}}=C_{t_{1}}$. Furthermore, consider the special case $\mathcal{S}_{1}=\mathcal{S}_{2}$. Then this in particular implies the equalities

$$
C_{s_{1}}=C_{t_{1}}=C_{s_{2}}=C_{t_{2}} .
$$

Hence, we obtain the assertion $C_{i}=C_{s}=C_{t}$ in condition (i), and the assertion $\operatorname{Supp} \mathcal{S}^{\prime}=C_{i}$ in condition (ii). The equalities in (4.6) and the rigidity of $\mathcal{S}_{1} \oplus \mathcal{S}_{2}$ also imply

$$
2=-c_{1}\left(\mathcal{S}_{1}\right) c_{1}\left(\mathcal{S}_{2}\right)=\chi_{S}\left(\mathcal{S}_{1}, \mathcal{S}_{2}\right)=\operatorname{dim} \operatorname{Hom}_{S}\left(\mathcal{S}_{1}, \mathcal{S}_{2}\right)+\operatorname{dim} \operatorname{Ext}_{S}^{2}\left(\mathcal{S}_{1}, \mathcal{S}_{2}\right) .
$$

Note that $\operatorname{Ext}_{S}^{2}\left(\mathcal{S}_{1}, \mathcal{S}_{2}\right) \cong \operatorname{Hom}_{S}\left(\mathcal{S}_{2}, \mathcal{S}_{1}\right)^{\vee}$ by Serre duality. 


\section{H. UEHARA}

(i) Now suppose $l\left(\mathcal{S}_{1}\right)>1$ and $l\left(\mathcal{S}_{2}\right)>1$. If $a_{s_{1}}>a_{t_{1}}$, there is a morphism

$$
\mathcal{S}_{1} \rightarrow \mathcal{O}_{C_{t_{1}}}\left(a_{t_{1}}\right)=\mathcal{O}_{C_{s_{1}}}\left(a_{t_{1}}\right) \hookrightarrow \mathcal{S}_{1}
$$

Hence, we have $\operatorname{dim} \operatorname{Hom}_{S}\left(\mathcal{S}_{1}, \mathcal{S}_{1}\right) \geqslant 2$, which contradicts (4.7) in the case $\mathcal{S}_{1}=\mathcal{S}_{2}$. Thus, we conclude that $a_{s_{1}}=a_{t_{1}}$ and $b_{s_{2}}=b_{t_{2}}$.

Similarly, if $a_{s_{1}} \neq b_{s_{2}}$, we see that

$$
\operatorname{dim} \operatorname{Hom}_{S}\left(\mathcal{S}_{1}, \mathcal{S}_{2}\right) \geqslant 4 \text { or } \operatorname{dim}_{\operatorname{Ext}_{S}^{2}}^{2}\left(\mathcal{S}_{1}, \mathcal{S}_{2}\right) \geqslant 4
$$

which again gives a contradiction to $(4.7)$.

(ii) Assume $b>a$. Then $\operatorname{dim} \operatorname{Hom}_{S}\left(\mathcal{S}, \mathcal{S}^{\prime}\right) \geqslant 4$. Similarly, if $b<a-1$, then $\operatorname{dim} \operatorname{Hom}_{S}\left(\mathcal{S}^{\prime}, \mathcal{S}\right) \geqslant 4$. Hence, both possibilities contradict (4.7), and we conclude that $b=a$ or $a-1$, as asserted.

(iii) Suppose $b=a$ in condition (ii). Then, by (4.7), we see that

$$
\operatorname{dim} \operatorname{Hom}_{S}\left(\mathcal{S}, \mathcal{S}^{\prime}\right)=2 \text { and } \operatorname{dim} \operatorname{Hom}_{S}\left(\mathcal{S}^{\prime}, \mathcal{S}\right)=0 .
$$

This implies the conclusion. Next, suppose $b=a-1$. Then, (4.7) implies

$$
\operatorname{dim} \operatorname{Hom}_{S}\left(\mathcal{S}, \mathcal{S}^{\prime}\right)=0 \text { and } \operatorname{dim}_{\operatorname{Hom}_{S}}\left(\mathcal{S}^{\prime}, \mathcal{S}\right)=2 .
$$

This implies the conclusion.

Let us proceed with a general statement for any $n$.

Lemma 4.9. Let $\alpha$ be a spherical object in $D_{Z_{0}}(S)$. Take elements

$$
\mathcal{S}_{1}:=\mathcal{S}_{s_{1}}\left(a_{s_{1}}, \ldots, a_{t_{1}}\right), \quad \mathcal{S}_{2}:=\mathcal{S}_{s_{2}}\left(b_{s_{2}}, \ldots, b_{t_{2}}\right) \in \Sigma(\alpha)
$$

(i) Let us take a reduced closed subscheme $Z^{\prime}=C_{i} \cup C_{i+1} \cup \cdots \cup C_{j}$ of $Z_{0}$ for some $i, j \in \mathbb{Z}$ with $0 \leqslant j-i \leqslant n-1$. Then $\left.\left(\mathcal{S}_{1} \oplus \mathcal{S}_{2}\right)\right|_{Z^{\prime}}$ is a rigid $\mathcal{O}_{S}$-module.

(ii) We have $C_{t_{1}} \neq C_{s_{2}-1}$.

(iii) For integers $l$ and $m$ satisfying $s_{1} \leqslant l \leqslant t_{1}$ and $s_{2} \leqslant m \leqslant t_{2}$ such that $C_{l}=C_{m}$, we have the following:

(1) $\left|a_{l}-b_{m}\right| \leqslant 1$.

(2) Suppose $s_{1}<l \leqslant t_{1}$ and $s_{2}=m \leqslant t_{2}$ :

A part of $\mathcal{S}_{1}$ :

The beginning of $\mathcal{S}_{2}$ :

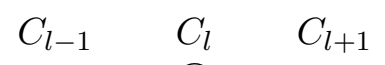

a ---

(bm) - - -

Then we have $a_{l} \geqslant b_{m}$.

(3) Suppose $s_{1}<l<t_{1}$ and $s_{2}=m=t_{2}$ :

$$
\begin{array}{cccc}
\text { A part of } \mathcal{S}_{1}: & C_{l-1} & C_{l} & C_{l+1} \\
\mathcal{S}_{2}: & & \text { a }_{l} & \\
& & \text { (b) } &
\end{array}
$$

Then we have $a_{l}=b_{m}+1$.

(4) Suppose $s_{1}<l=t_{1}$ and $s_{2}=m<t_{2}$ :

The end of $\mathcal{S}_{1}$ : $C_{l-1} \quad C_{l} \quad C_{l+1}$

The beginning of $\mathcal{S}_{2}$ :

Then we have $a_{l}=b_{m}$. 


\section{Autoequivalences of Elliptic surfaces}

Proof. (i) We may assume $Z^{\prime} \neq Z$. Let us consider the restriction map

$$
\left.S_{1} \oplus \mathcal{S}_{2} \rightarrow\left(\mathcal{S}_{1} \oplus \mathcal{S}_{2}\right)\right|_{Z^{\prime}}
$$

Note that there are no homomorphisms from its kernel to $\left.\left(\mathcal{S}_{1} \oplus \mathcal{S}_{2}\right)\right|_{Z^{\prime}}$, since their supports intersect at finitely many points. Then we can apply Mukai's lemma (see [KO94, Lemma 2.2(2)]), and hence the rigidity of $\mathcal{S}_{1} \oplus \mathcal{S}_{2}$ implies the rigidity of $\left.\left(\mathcal{S}_{1} \oplus \mathcal{S}_{2}\right)\right|_{Z^{\prime}}$ as an $\mathcal{O}_{S}$-module.

(ii) Assume to the contrary that $C_{t_{1}}=C_{s_{2}-1}$. Then we can deduce a contradiction in a way completely similar to the one in the proof of $C_{s}=C_{t}$ in Lemma 4.8(i).

(iii) Take $Z^{\prime}=C_{l}$ in assertion (i) for statement (1), and $Z^{\prime}=C_{l-1} \cup C_{l}$ in assertion (i) for statement (2) in the case $n>2$. Then statement (1) follows immediately from equation (2.2) in Section 2.2 and the rigidity of

$$
\mathcal{O}_{C_{l}}\left(a_{l}\right) \oplus \mathcal{O}_{C_{m}}\left(b_{m}\right)=\mathcal{O}_{C_{l}}\left(a_{l}\right) \oplus \mathcal{O}_{C_{l}}\left(b_{m}\right),
$$

which is a direct summand of the rigid sheaf $\left.\left(\mathcal{S}_{1} \oplus \mathcal{S}_{2}\right)\right|_{C_{l}}$. In the situation of statement (2) in the case $n>2$, we know the rigidity of

$$
\mathcal{O}_{C_{l-1} \cup C_{l}}\left(a_{l-1}, a_{l}\right) \oplus \mathcal{O}_{C_{m}}\left(b_{m}\right)=\mathcal{O}_{C_{l-1} \cup C_{l}}\left(a_{l-1}, a_{l}\right) \oplus \mathcal{O}_{C_{l}}\left(b_{m}\right) .
$$

Then, there is a surjection

$$
\operatorname{Hom}_{S}\left(\mathcal{O}_{C_{l}}\left(b_{m}\right), \mathcal{O}_{C_{l}}\left(a_{l}\right)\right) \rightarrow \operatorname{Ext}_{S}^{1}\left(\mathcal{O}_{C_{l}}\left(b_{m}\right), \mathcal{O}_{C_{l-1}}\left(a_{l-1}-1\right)\right) .
$$

The non-vanishing of the latter space forces the result. Statement (2) in the case $n=2$ is proved in Lemma 4.8.

We leave it to the reader to show statement (3), since all necessary ideas have already appeared. Statement (4) is a direct consequence of statement (2).

\section{The proof of Proposition 4.4}

Suppose that we are given a spherical object $\alpha \in D_{Z_{0}}(S)$ with $l(\alpha)=1$. Then we get $\alpha \cong$ $\mathcal{O}_{C_{b}}(a)[i]$ for some $a, b, i \in \mathbb{Z}$. (Or, assume that $l(\alpha)<n$. This condition implies $\operatorname{Supp} \alpha \neq Z_{0}$, and then [IU05, Proposition 1.6] yields Proposition 4.4.) If we prove that for a spherical $\alpha$ with $l(\alpha)>1$, there is an autoequivalence $\Psi \in B_{0}$ such that

$$
l(\Psi(\alpha))<l(\alpha),
$$

then, since $\Psi(\alpha)$ is again spherical, induction on $l(\alpha)$ yields Proposition 4.4. On the other hand, for every $\Psi \in$ Auteq $D_{Z_{0}}(S)$, we have the inequality

$$
l(\Psi(\alpha)) \leqslant \sum_{q} l\left(\Psi\left(H^{q}(\alpha)\right)\right)
$$

(the proof is the same as that of [IU05, Lemma 4.11]). Thus, to get (5.1), it is enough to show

$$
\sum_{q} l\left(\Psi\left(H^{q}(\alpha)\right)\right)<\sum_{q} l\left(H^{q}(\alpha)\right)(=l(\alpha)) .
$$

\subsection{Auxiliary results}

Let us begin with the following lemma.

Lemma 5.1. Take integers $s$ and $t$ with $s \leqslant t$, a complex analytic open subset $U=U_{s, \ldots, t}$ and $\Psi \in\left\langle T_{\mathcal{O}_{C_{i}}(a)} \mid s \leqslant i \leqslant t, a \in \mathbb{Z}\right\rangle$. Let $\beta$ be an object of $D_{Z_{0}}(S)$. Then we have the following: 


\section{H. UEHARA}

(i) $\left.\Psi(\beta)\right|_{U} \cong \Psi\left(\left.\beta\right|_{U}\right)$.

(ii) $l(\Psi(\beta))-l(\beta)=l\left(\left.\Psi(\beta)\right|_{U}\right)-l\left(\left.\beta\right|_{U}\right)$.

Proof. It is enough to consider the case $\Psi=T_{\mathcal{O}_{C}(a)}$ for some $a$ and some $C=C_{i}$ with $s \leqslant i \leqslant t$.

(i) Since $\operatorname{Supp} \mathcal{O}_{C}(a)=C \subset U$, we have

$$
\mathbb{R H o m}_{D(S)}\left(\mathcal{O}_{C}(a), \beta\right) \cong \mathbb{R} \operatorname{Hom}_{D(U)}\left(\mathcal{O}_{C}(a),\left.\beta\right|_{U}\right) .
$$

Hence, there is the isomorphism of exact triangles in $D(U)$ :

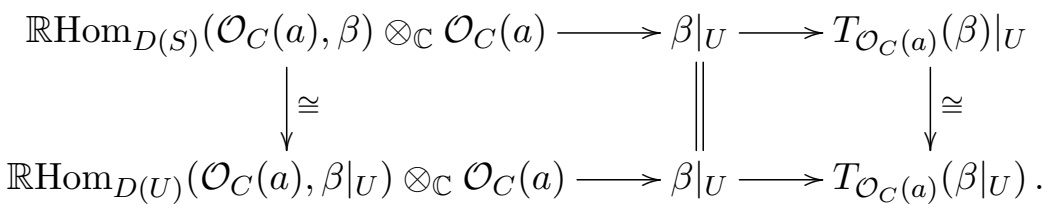

(ii) From the exact triangle (2.3), it is easy to see the equality

$$
\left.\left(T_{\mathcal{O}_{C}(a)}(\beta)\right)\right|_{S \backslash C}=\left.\beta\right|_{S \backslash C} .
$$

Hence we have

$$
\sum_{i: U \cap C_{i}=\emptyset} l_{i}\left(T_{\mathcal{O}_{C}(a)}(\beta)\right)=\sum_{i: U \cap C_{i}=\emptyset} l_{i}(\beta)
$$

Since by the definition of $l\left(\left.\beta\right|_{U}\right)$ we have

$$
l(\beta)=l\left(\left.\beta\right|_{U}\right)+\sum_{i: U \cap C_{i}=\emptyset} l_{i}(\beta),
$$

we obtain

$$
l\left(T_{\mathcal{O}_{C}(a)}(\beta)\right)-l(\beta)=l\left(\left.\left(T_{\mathcal{O}_{C}(a)}(\beta)\right)\right|_{U}\right)-l\left(\left.\beta\right|_{U}\right)
$$

as desired.

By Lemma 5.1(i), we can use computations of [IU05, Lemma 4.15] in our setting. For example, when $n=3$, taking $U=U_{0,1}$ and $U_{1,2}$ in Lemma 5.1(i), we have $T_{\mathcal{O}_{C_{1}}(-1)}\left(\mathcal{S}_{C_{1} \cup C_{2} \cup C_{3} \cup C_{1}}(0,0,0,0)\right)$ $=\mathcal{O}_{C_{2} \cup C_{3}}$.

Lemma 5.1(ii) is useful to prove (5.2) as it allows us to apply many results from the $A_{n}$-case of [IU05] to our $\tilde{A}_{n}$-case. Namely, let $\mathcal{S} \in \Sigma(\alpha)$ and $U=U_{s, \ldots, t}$ be as above. Then there is a smooth surface $\hat{S}$ containing an $A_{t-s+3}$-configuration $\hat{Z}_{0}$ and an open subset $\hat{U} \cong U$ such that $\hat{U} \cap \hat{Z}_{0} \cong U \cap Z$, together with a sheaf $\hat{\mathcal{S}}$ on $\hat{S}$ such that $\left.\left.\hat{\mathcal{S}}\right|_{\hat{U}} \cong \mathcal{S}\right|_{U}$. Now, applying Lemma 5.1(ii) twice, we get the equality

$$
l(\Psi(\mathcal{S}))-l(\mathcal{S})=l(\Psi(\hat{\mathcal{S}}))-l(\hat{\mathcal{S}}) .
$$

The right-hand side of this equation is computed in many cases in [IU05]; see in particular [IU05, Lemma 4.15]. In the following, we will often refer the reader to statements in [IU05] and claim that the proof in our case is analogous. Note that in many steps of the proof in [IU05], we referred to [IU05, (6.2)] and left the details to the reader. Many of the details (or rather their analogues in the $\tilde{A}_{n}$-case) are stated explicitly in Lemmas 4.8 and 4.9 of the present paper.

To prove Lemma A below, we need local versions of [IU05, Lemmas 6.3 and 6.6].

Lemma 5.2 (cf. [IU05, Lemma 6.3]). Let $\alpha \in D_{Z_{0}}(S)$ be a spherical object and $C=C_{s} \subset Z_{0}$ a (-2)-curve. Take a sufficiently small complex analytic neighbourhood $U=U_{s}$ of $C$. Assume 


\section{Autoequivalences of Elliptic surfaces}

that for every $p$ we have a decomposition

$$
\left.H^{p}(\alpha)\right|_{U}=\bigoplus_{j}^{r_{1}^{p}} \mathcal{R}_{1, j}^{p} \oplus \bigoplus_{j}^{r_{2}^{p}} \mathcal{R}_{2, j}^{p} \oplus \bigoplus_{j}^{r_{3}^{p}} \mathcal{R}_{3, j}^{p} \oplus \bigoplus_{j}^{r_{4}^{p}} \mathcal{R}_{4, j}^{p},
$$

where the $\mathcal{R}_{k, j}^{p}$ are sheaves of the following forms:

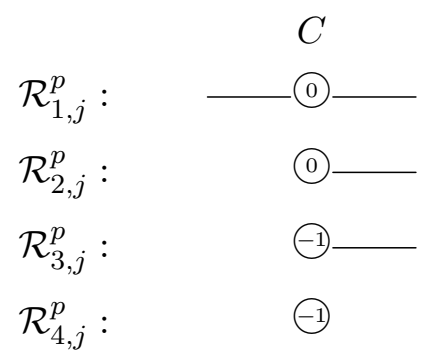

In this situation, we have the following:

(i) If $\sum_{p} r_{2}^{p}>\sum_{p} r_{3}^{p}$, then $l\left(T_{\mathcal{O}_{C}(-1)}(\alpha)\right)<l(\alpha)$.

(ii) If $\sum_{p} r_{2}^{p}<\sum_{p} r_{3}^{p}$, then $l\left(T_{\mathcal{O}_{C}(-2)}(\alpha)\right)<l(\alpha)$.

Proof. The assumption in statement (i) and [IU05, Lemma 4.15] imply the inequality

$$
\sum_{p} l\left(T_{\mathcal{O}_{C}(-1)}\left(\left.H^{p}(\alpha)\right|_{U}\right)\right)<\sum_{p} l\left(\left.H^{p}(\alpha)\right|_{U}\right)\left(=l\left(\left.\alpha\right|_{U}\right)\right) .
$$

Combining this with Lemma 5.1, we obtain inequality (5.2) for $\Psi=T_{\mathcal{O}_{C}(-1)}$. Then the desired result follows as explained above.

The proof of statement (ii) is similar.

LEMma 5.3 (cf. [IU05, Lemma 6.6]). Under the assumptions of Lemma 5.2, assume that $\sum_{p} r_{2}^{p}=$ $\sum_{p} r_{3}^{p} \neq 0$ holds. Then $r_{4}^{p}=0$ for all $p$.

Proof. According to the decomposition of $\left.H^{p}(\alpha)\right|_{U}$ in Lemma 5.2, we can decompose $H^{p}(\alpha)$ as

$$
H^{p}(\alpha)=\bigoplus_{j^{\prime}} \widetilde{\mathcal{R}_{1, j^{\prime}}^{p}} \oplus \bigoplus_{j}^{r_{2}^{p}} \widetilde{\mathcal{R}_{2, j}^{p}} \oplus \bigoplus_{j}^{r_{3}^{p}} \widetilde{\mathcal{R}_{3, j}^{p}} \oplus \bigoplus_{j}^{r_{4}^{p}} \mathcal{R}_{4, j}^{p},
$$

where $\widetilde{\mathcal{R}_{1, j^{\prime}}^{p}}$ satisfies $s\left(\widetilde{\mathcal{R}_{1, j^{\prime}}^{p}}\right) \neq s$, and $\widetilde{\mathcal{R}_{k, j}^{p}}(k=2,3)$ is a sheaf in $\Sigma(\alpha)$ such that $\mathcal{R}_{k, j}^{p}$ is a direct summand of $\left.\widetilde{\mathcal{R}_{k, j}^{p}}\right|_{U}$. Here, note that $\left.\widetilde{\mathcal{R}_{2, j}^{p}}\right|_{U}$ and $\left.\widetilde{\mathcal{R}_{3, j}^{p}}\right|_{U}$ may possibly contain several direct summands of the form $\mathcal{R}_{1, j}^{p}$.

Applying the proof of [IU05, Lemma 6.6] for this decomposition, we obtain the assertion. Note that in the proof we use [IU05, Lemmas 6.4 and 6.5]. However, our setting requires some slight changes. For example, we should replace the notation $X$ and $Z$ with $S$ and $Z_{0}$, respectively, and the vertical arrows of [IU05, diagram (6.3)] are not isomorphisms anymore. Instead, one is injective and the other surjective. Hence, we define $\bar{\eta}^{p}$ to be the following composition:

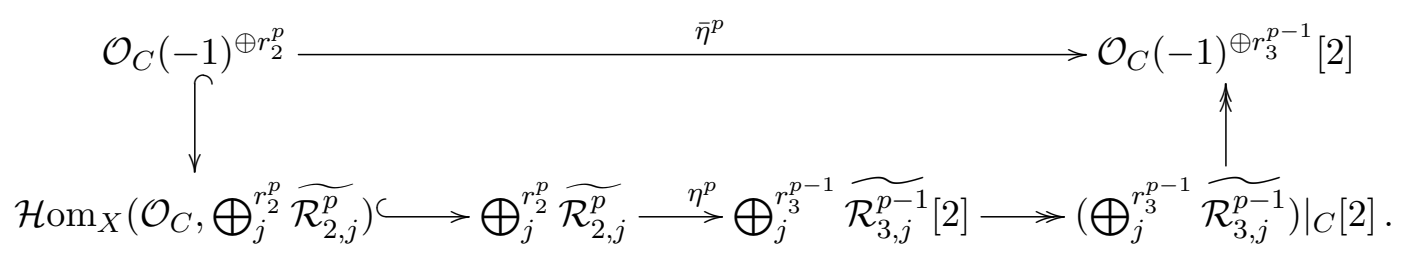




\section{H. UEHARA}

Here the right vertical arrow is the projection to the direct summand.

Lemma A (cf. [IU05, Lemma A]). Let $\alpha \in D_{Z_{0}}(S), U=U_{s}$ and $C=C_{s}$ be as above. Assume that we can write

$$
\left.\bigoplus_{p} \mathcal{H}^{p}(\alpha)\right|_{U}=\bigoplus_{j}^{r_{1}} \mathcal{R}_{1, j} \oplus \bigoplus_{j}^{r_{2}} \mathcal{R}_{2, j} \oplus \bigoplus_{j}^{r_{3}} \mathcal{R}_{3, j} \oplus \bigoplus_{j}^{r_{4}} \mathcal{R}_{4, j}
$$

such that the $\mathcal{R}_{k, j}$ are elements of $\Sigma(\alpha)_{U}$ and are of the following forms:

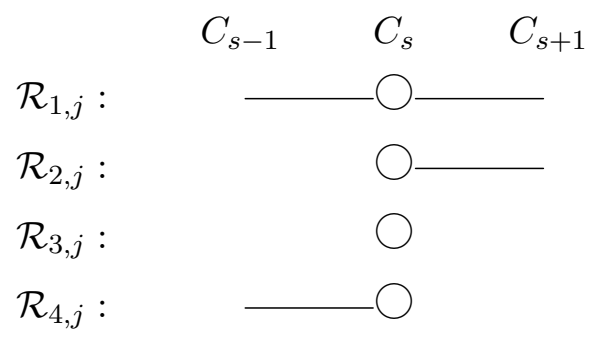

Suppose that either $r_{3} \neq 0$ or $r_{2} \cdot r_{4} \neq 0$ holds, and suppose furthermore that $\operatorname{Supp} \alpha \neq C$. Then, there is an integer a such that $l\left(T_{\mathcal{O}_{C}(a)}(\alpha)\right)<l(\alpha)$.

Proof. The proof goes parallel to that of [IU05, Lemma A]. Let us denote by $\widetilde{\mathcal{R}_{1, j}}$ an element in $\Sigma(\alpha)$ such that $\mathcal{R}_{1, j}$ is a direct summand of $\left.\widetilde{\mathcal{R}_{1, j}}\right|_{U}$.

When $r_{2}=r_{4}=0$, we can see that

$$
\chi\left(\widetilde{\mathcal{R}_{1, j}}, \mathcal{R}_{3, k}\right)=0
$$

for any $j$ and $k$. Note that [IU05, Lemma 6.2] is true without any changes in our situation, so that we can conclude $r_{1}=0$. This contradicts the assumption $\operatorname{Supp} \alpha \neq C$. Therefore, by symmetry, we may safely assume $r_{2} \neq 0$.

In the case $r_{2} \cdot r_{4} \neq 0$, we can see from Lemma 4.9 that there is an integer $a$ such that

$$
\operatorname{deg}_{C} \mathcal{R}_{2, h}=\operatorname{deg}_{C} \mathcal{R}_{4, k}=a
$$

for all $h$ and $k$, and that $\operatorname{deg}_{C} \mathcal{R}_{3, j}$ is $a$ or $a-1$. Then by Lemma 5.1(ii) and [IU05, Lemma 4.15], inequality (5.2) holds for $\Psi=T_{\mathcal{O}_{C}(a-1)}$. Hence, we obtain the desired result.

What remains is the case $r_{2} \cdot r_{3} \neq 0$ and $r_{4}=0$. First, suppose that

$$
\operatorname{deg}_{C_{s}} \mathcal{R}_{3, h}=\max _{k, j} \operatorname{deg}_{C_{s}} \mathcal{R}_{k, j}(=: b)
$$

holds for some $h$. Then $\operatorname{deg}_{C_{s}} \mathcal{R}_{1, j}=\operatorname{deg}_{C_{s}} \mathcal{R}_{2, j}=b$ for all $j$, and if we put $\Psi=T_{\mathcal{O}_{C}(b-1)}$, again using Lemma 5.1(ii) along with [IU05, Lemma 4.15], we obtain inequality (5.2).

Next, suppose that $\operatorname{deg}_{C_{s}} \mathcal{R}_{3, j}=b-1$ holds for all $j$. In this case, just apply Lemmas 5.2 and 5.3, which imply the assertion.

Lemma B (cf. [IU05, Lemma B]). Let $\alpha \in D_{Z_{0}}(S)$ be a spherical object and fix positive integers $s$ and $t$ with $1 \leqslant t-s \leqslant n-2$. Take a sufficiently small complex analytic neighbourhood $U=U_{s, \ldots, t}$ of $C_{s} \cup \cdots \cup C_{t}$ and assume that we can write

$$
\left.\bigoplus_{p} H^{p}(\alpha)\right|_{U}=\bigoplus_{j}^{r_{1}} \mathcal{R}_{1, j} \oplus \bigoplus_{j}^{r_{2}} \mathcal{R}_{2, j} \oplus \bigoplus_{j}^{r_{3}} \mathcal{R}_{3, j} \oplus \bigoplus_{j}^{r_{4}} \mathcal{R}_{4, j},
$$




\section{Autoequivalences of Elliptic surfaces}

where the $\mathcal{R}_{k, j}$ are elements of $\Sigma(\alpha)_{U}$ and are of the following forms:

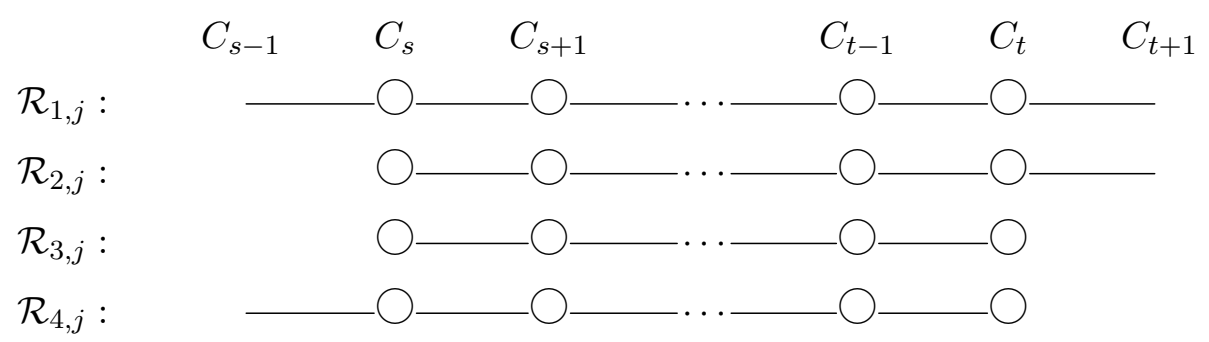

Suppose that either $r_{3} \neq 0$ or $r_{2} \cdot r_{4} \neq 0$ holds. Then there is a

$$
\Phi \in\left\langle T_{\mathcal{O}_{C_{l}}(a)} \mid a \in \mathbb{Z}, s \leqslant l \leqslant t\right\rangle
$$

such that $l(\Phi(\alpha))<l(\alpha)$.

Proof. The proof goes parallel to that of [IU05, Lemma B]. Note that the straight-forward analogues of [IU05, Lemmas 6.7 and 6.8] hold for $\left.H^{p}(\alpha)\right|_{U}$, and use them.

\subsection{The proof of Proposition 4.4}

Notice that if we show the existence of an autoequivalence $\Phi \in B_{0}$ such that $l(\alpha)>l(\Phi(\alpha))$ under the assumption $l(\alpha)>1$, then we can prove the statement by induction on $l(\alpha)$. Recall that the proof is already done in [IU05, Proposition 1.7] in the case Supp $\alpha \neq Z_{0}$, since in that case $\alpha$ is supported by a chain of projective lines contained in $Z_{0}$. Hence, we may assume $\operatorname{Supp} \alpha=Z_{0}=C_{1} \cup \cdots \cup C_{n}$.

For $\mathcal{S}=\mathcal{S}_{s}\left(a_{s}, \ldots, a_{t}\right) \in \Sigma(\alpha)$, define an integer $s(\mathcal{S})$ by the properties

$$
C_{s(\mathcal{S})}=C_{s} \text { and } \quad 1 \leqslant s(\mathcal{S}) \leqslant n
$$

and an integer $t(\mathcal{S})$ by the properties

$$
C_{t(\mathcal{S})}=C_{t} \quad \text { and } \quad s(\mathcal{S}) \leqslant t(\mathcal{S}) \leqslant s(\mathcal{S})+n-2 .
$$

Here, note that Lemma 4.9(ii) guarantees that for $\mathcal{R} \in \Sigma(\alpha)$, there are no elements $\mathcal{S} \in \Sigma(\alpha)$ such that $C_{t(\mathcal{S})}=C_{s(\mathcal{R})-1}$ or $C_{t(\mathcal{R})}=C_{s(\mathcal{S})-1}$. Thus, we have

$$
l_{s(\mathcal{R})-1}(\alpha)<l_{s(\mathcal{R})}(\alpha) \text { and } \quad l_{t(\mathcal{R})}(\alpha)>l_{t(\mathcal{R})+1}(\alpha) .
$$

Therefore, we can find integers $s_{0}$ and $t_{0}$ such that

$$
l_{s_{0}-1}(\alpha)<l_{s_{0}}(\alpha)=l_{s_{0}+1}(\alpha)=\cdots=l_{t_{0}}(\alpha)>l_{t_{0}+1}(\alpha) .
$$

Then we are in the situation of Lemma A (if $s_{0}=t_{0}$ ) or Lemma B (if $s_{0}<t_{0}$ ). So the proof is done.

Remark 5.4. Take an arbitrary element $\mathcal{R} \in \Sigma(\alpha)$. Then, in the proof above, we can find $s_{0}$ and $t_{0}$ such that $s(\mathcal{R}) \leqslant s_{0} \leqslant t_{0} \leqslant t(\mathcal{R})$. Thus, Lemma $\mathrm{A}$ or B provides

$$
\Phi \in\left\langle T_{\mathcal{O}_{C_{l}}(a)} \mid a \in \mathbb{Z}, s(\mathcal{R}) \leqslant l \leqslant t(\mathcal{R})\right\rangle
$$

such that $l(\alpha)>l(\Phi(\alpha))$. We shall use this remark in Section 6 . 


\section{H. UEHARA}

\section{The proof of Proposition 4.2}

\subsection{Facts needed for the proof of Proposition 4.2}

Let $\Phi$ be an autoequivalence of $D_{Z_{0}}(S)$ that preserves the cohomology class of a point. Put $\alpha=\Phi\left(\mathcal{O}_{C_{1}}\right)$ and $\beta=\Phi\left(\mathcal{O}_{C_{1}}(-1)\right)$. Applying Proposition 4.4 and the shift functor [1], we may assume

$$
\alpha=\mathcal{O}_{C_{b}}(a)
$$

for some $a, b \in \mathbb{Z}$ with $1 \leqslant b \leqslant n$. To prove Proposition 4.2 , it suffices to show the following.

Claim 6.1. Suppose $l(\beta)>1$. There is an autoequivalence $\Psi \in B_{0}$ such that $l(\Psi(\alpha))=1$ and $l(\beta)>l(\Psi(\beta))$.

In fact, Proposition 4.2 easily follows from this.

Proof of Proposition 4.2. By Claim 6.1, we can reduce the problem to the case $l(\alpha)=l(\beta)=1$. In this case, the supports of $\alpha$ and $\beta$ must be the same by Fact 6.5 below. Therefore, we get the conclusion from the $A_{1}$-case [IU05, Proposition 1.6], and we can complete the proof of Proposition 4.2 by induction on $l(\beta)$.

Most of the proof of Claim 6.1 goes parallel to that of [IU05, Claim 7.1].

FACT 6.2 (cf. [IU05, Condition 7.2]). We may assume

$$
\max \left\{\operatorname{deg}_{C_{b}} \mathcal{R} \mid \mathcal{R} \in \Sigma(\beta)_{U_{b}}, \operatorname{Supp} \mathcal{R} \supset C_{b}\right\}=0 .
$$

In particular, $\operatorname{deg}_{C_{b}} \mathcal{R}=0$ or -1 for all $\mathcal{R} \in \Sigma(\beta)_{U_{b}}$ with Supp $\mathcal{R} \supset C_{b}$ by Lemma 4.9(iii)(1).

The relations between $\mathcal{O}_{C_{1}}$ and $\mathcal{O}_{C_{1}}(-1)$ impose conditions on $a$ and $\beta$. From the spectral sequence

$$
E_{2}^{p, q}=\operatorname{Ext}_{S}^{p}\left(H^{-q}(\beta), \mathcal{O}_{C_{b}}(a)\right) \Longrightarrow \operatorname{Hom}_{D(S)}^{p+q}(\beta, \alpha)= \begin{cases}\mathbb{C}^{2}, & p+q=0 \\ 0, & p+q \neq 0\end{cases}
$$

we obtain the following result.

FACT 6.3 (cf. [IU05, Condition 7.3]). We have $E_{2}^{1, q}=0$ for $q \neq-1$.

FACT 6.4 (cf. [IU05, Condition 7.4]). The map $d_{2}^{0,-1}: E_{2}^{0,-1} \rightarrow E_{2}^{2,-2}$ is injective, $d_{2}^{0,0}: E_{2}^{0,0} \rightarrow$ $E_{2}^{2,-1}$ is surjective, and the $d_{2}^{0, q}: E_{2}^{0, q} \rightarrow E_{2}^{2, q+1}$ are isomorphisms for all $q \neq 0,-1$.

In addition to Facts 6.3 and 6.4, the spectral sequence (6.1) implies

$$
\operatorname{dim} \text { Coker } d_{2}^{0,-1}+\operatorname{dim} \operatorname{Ker} d_{2}^{0,0}+\operatorname{dim} E_{2}^{1,-1}=2 .
$$

To show the following, we need the assumption that $\Phi$ preserves the cohomology class $\operatorname{ch}\left(\mathcal{O}_{x}\right)$ in $H^{4}(S, \mathbb{Q}) .^{3}$

FACT 6.5 (cf. [IU05, Condition 7.5]). The equality $c_{1}(\beta)=\left[C_{b}\right]$ holds in the cohomology group $H^{2}(S, \mathbb{Q})$.

${ }^{3}$ If we drop this assumption, we can just conclude that $c_{1}(\beta)=\left[C_{b}\right]+k\left[Z_{0}\right]$ for some $k \in \mathbb{Z}$ by a proof similar to that of Condition 7.5 in [IU05]. 


\section{Autoequivalences of Elliptic surfaces}

Proof. By the assumption on $\Phi$, we have

$$
0=c_{1}\left(\mathcal{O}_{x}\right)=c_{1}\left(\Phi\left(\mathcal{O}_{x}\right)\right)=c_{1}(\alpha)-c_{1}(\beta),
$$

which gives the assertion.

Claim 6.6 (cf. [IU05, Claim 7.6]). We have $a \geqslant-1$.

Proof. The proof is similar to that of [IU05, Claim 7.6].

Claim 6.7 (cf. [IU05, Claim 7.7]). Fix $q \neq 0$. If $E_{2}^{2,-q-1}=0$ in (6.1), then we have $\operatorname{deg}_{C_{b}} \mathcal{R}>a$ for all direct summands $\mathcal{R} \in \Sigma(\beta)_{U_{b}}$ of $\left.H^{q}(\beta)\right|_{U_{b}}$ with $\operatorname{Supp} \mathcal{R} \supset C_{b}$. If, in addition, we suppose $a \geqslant 0$, then we get $C_{b} \not \subset \operatorname{Supp} H^{q}(\beta)$.

Proof. The proof of Claim 6.7 is similar to that of [IU05, Claim 7.7].

Remark 6.8. Below, we use the notation $t(\mathcal{R})$ and $s(\mathcal{R})$ for $\mathcal{R} \in \Sigma(\beta)$ defined in Section 5.2. Recall that $b$ satisfies $1 \leqslant b \leqslant n$ by definition.

If there is an element $\mathcal{R} \in \Sigma(\beta)$ with

$$
\text { either }(\mathcal{R})-n+1<b<s(\mathcal{R})-1 \text { or } t(\mathcal{R})+1<b \text {, }
$$

then we can find $\Psi \in\left\langle T_{\mathcal{O}_{C_{l}}(a)} \mid a \in \mathbb{Z}, C_{l} \subset \operatorname{Supp} \mathcal{R}\right\rangle$ such that $\Psi(\alpha) \cong \alpha$ and $l(\Psi(\beta))<l(\beta)$ by Remark 5.4. Therefore, we may assume

$$
s(\mathcal{R})-1 \leqslant b \leqslant t(\mathcal{R})+1 \quad \text { or } \quad b+n \leqslant t(\mathcal{R})+1
$$

for all $\mathcal{R} \in \Sigma(\beta)$.

\subsection{Division into cases}

Now, we divide the proof into cases as in [IU05, 7.2, Division into cases, p. 426]. We only have to consider three:

(i) We have $C_{b} \subset \operatorname{Supp} \mathcal{R}$ for all $\mathcal{R} \in \Sigma(\beta)_{U_{b}}$.

(ii) There is an $\mathcal{R} \in \Sigma(\beta)_{U_{b}}$ with $\operatorname{Supp} \mathcal{R} \cap C_{b}=C_{b+1} \cap C_{b}$, but there is no $\mathcal{R}^{\prime} \in \Sigma(\beta)_{U_{b}}$ with $\operatorname{Supp} \mathcal{R}^{\prime} \cap C_{b}=C_{b-1} \cap C_{b}$.

(iii) There are $\mathcal{R}, \mathcal{R}^{\prime} \in \Sigma(\beta)_{U_{b}}$ with $\operatorname{Supp} \mathcal{R} \cap C_{b}=C_{b+1} \cap C_{b}$ and $\operatorname{Supp} \mathcal{R}^{\prime} \cap C_{b}=C_{b-1} \cap C_{b}$.

Case (i). In this case, we can find $\Psi$ in Claim 6.1 in a way similar to that of [IU05, Section 7.3, Case (i)], after some obvious changes; for instance, [IU05, Claim 7.8] should be replaced by the following.

Claim 6.9. $\left.\mathcal{O}_{\ldots \cup C_{b}}(*,-1)\right|_{U_{b}},\left.\mathcal{O}_{C_{b} \cup \ldots}(-1, *)\right|_{U_{b}},\left.\mathcal{O}_{\ldots \cup C_{b} \cup \ldots(*,-1, *)}\right|_{U_{b}} \notin \Sigma(\beta)_{U_{b}}$.

Case (ii). The existence of $\mathcal{R} \in \Sigma(\beta)_{U_{b}}$ with $\operatorname{Supp} \mathcal{R} \cap C_{b}=C_{b} \cap C_{b+1}$ and Lemma 4.9(ii) imply the non-existence of $\mathcal{S} \in \Sigma(\beta)_{U_{b}}$ with $\operatorname{Supp} \mathcal{S} \cap C_{b+1}=C_{b} \cap C_{b+1}$. Note that $n>2$ in this case by Lemma 4.8(i). Furthermore, we have

$$
\Sigma(\beta)_{U_{b}} \subset\left\{\left.\mathcal{O}_{C_{b} \cup \ldots}\left(a^{\prime}, *\right)\right|_{U_{b}},\left.\mathcal{O}_{C_{b+1}}(*)\right|_{U_{b}},\left.\mathcal{O}_{\ldots \cup C_{b} \cup \ldots} \cup\left(*, a^{\prime}, *\right)\right|_{U_{b}} \mid a^{\prime}=-1,0\right\} .
$$

Now, we can find $\Psi$ in Claim 6.1 in a way similar to that of [IU05, Case (ii)].

Case (iii). Fact 6.3 implies that $\mathcal{R}$ and $\mathcal{R}^{\prime}$ above must be in $H^{1}(\beta)$. Moreover, they are unique in a decomposition of $H^{1}(\beta)$, by virtue of the inequality $\operatorname{dim} E_{2}^{1,-1} \leqslant 2$ from (6.2). 


\section{H. UEHARA}

We can exclude the case $n=2$ as follows. Suppose $n=2$. Recall that $\mathcal{R}$ is the unique element in $\Sigma(\beta)$ such that $C_{s(\mathcal{R})} \neq C_{b}$, and $\mathcal{R}^{\prime}$ is the unique element in $\Sigma(\beta)$ such that $C_{t\left(\mathcal{R}^{\prime}\right)} \neq C_{b}$. It follows from Lemma 4.8(i) that every element $\mathcal{S} \in \Sigma(\beta)$ satisfies $C_{s(\mathcal{S})}=C_{t(\mathcal{S})}$. Therefore $\beta[-1]$ is an indecomposable sheaf $\mathcal{S}\left(=\mathcal{R}=\mathcal{R}^{\prime}\right)$ satisfying $C_{s(\mathcal{S})}=C_{t(\mathcal{S})} \neq C_{b}$. In this case, Fact 6.5 cannot be satisfied.

Now, Lemma 4.9 allows us to write

$$
\left.\bigoplus_{p} H^{p}(\beta)\right|_{U_{b-1, b}}=\bigoplus_{j}^{r_{1}} \mathcal{R}_{1, j} \oplus \bigoplus_{j}^{r_{2}} \mathcal{R}_{2, j} \oplus \mathcal{R}_{3} \oplus \mathcal{R}_{4},
$$

where the $\mathcal{R}_{k, j}$ and $\mathcal{R}_{3}$ and $\mathcal{R}_{4}$ are sheaves in $\Sigma(\beta)_{U_{b-1, b}}$ of the following forms:

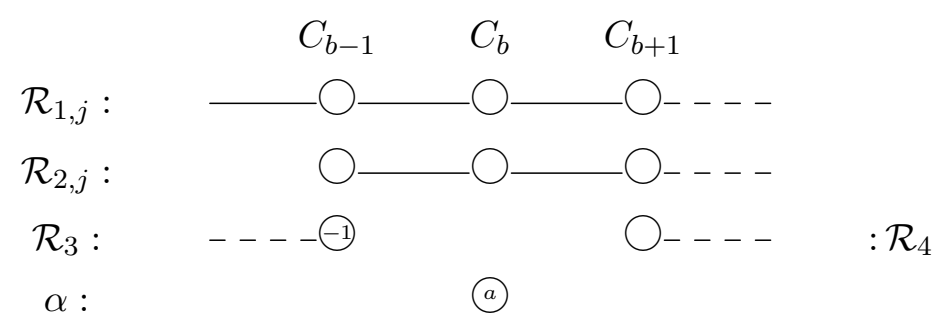

Here, we assume $\operatorname{deg}_{C_{b-1}} \mathcal{R}_{3}=-1$, for simplicity.

Claim 6.10. We have $a=-1$.

Proof. A proof similar to that of [IU05, Claim 7.11] works.

The inequality $\operatorname{dim} E_{2}^{1,-1} \leqslant 2$ from (6.2) also implies

$$
\operatorname{Ext}_{S}^{1}\left(\mathcal{R}_{k, j}, \mathcal{O}_{C_{b}}(-1)\right)=0
$$

for $k=1,2$ and for all $j$. In particular, we get

$$
\operatorname{deg}_{C_{b}} \mathcal{R}_{1, j}=\operatorname{deg}_{C_{b}} \mathcal{R}_{2, j}=0 .
$$

Recall that there is a unique sheaf $\mathcal{R} \in \Sigma(\beta)$ satisfying $C_{t(\mathcal{R})}=C_{b-1}$, and that $\left.\mathcal{R}\right|_{U_{b-1, b}}$ contains $\mathcal{R}_{3}$ as a direct summand. Now, we give a proof for case (iii) by induction on $l(\mathcal{R})$.

First, suppose $l(\mathcal{R})=1$. In this case $\mathcal{R}=\mathcal{R}_{3}$, and we write

$$
\bigoplus_{j}^{r_{2}} \mathcal{R}_{2, j}=\bigoplus_{j}^{s_{1}} \mathcal{S}_{1, j} \oplus \bigoplus_{j}^{s_{2}} \mathcal{S}_{2, j}
$$

where the $\mathcal{S}_{k, j}$ are sheaves in $\Sigma(\beta)_{U_{b-1, b}}$ of the following forms:

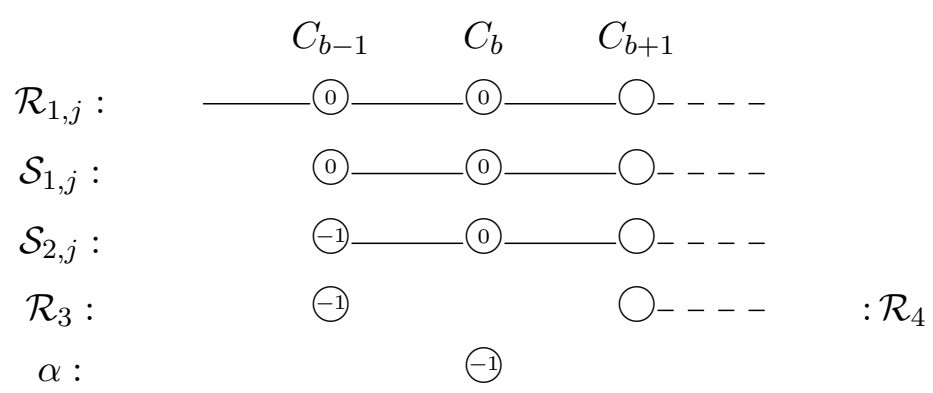

Note that we may assume $r_{1} \neq 0$ since otherwise $\operatorname{Supp} \beta$ is strictly smaller than $Z_{0}$, in which case [IU05, Claim 7.1] gives the result. Because of the existence of $\mathcal{R}_{3}$, we have $r_{2} \neq 0$ by [IU05, 


\section{Autoequivalences of Elliptic surfaces}

Lemma 6.2], which continues to hold in our setting. Hence, $s_{1} \neq s_{2}$ by Lemma 5.3. Define

$$
\Psi_{0}= \begin{cases}T_{\mathcal{O}_{C_{b-1}} \cup C_{b}}(-1,-1) & \text { if } s_{1}<s_{2}, \\ T_{\mathcal{O}_{C_{b-1}} \cup C_{b}}^{\prime} & \text { if } s_{2}<s_{1} .\end{cases}
$$

Then, as one can see using the computations of [IU05, Lemma 4.15], the pair $\left(\Psi_{0}(\alpha), \Psi_{0}(\beta)\right)$ fits in case (ii) and $\Psi_{0}(\beta)$ satisfies $l\left(\Psi_{0}(\beta)\right) \leqslant l(\beta)$. Since we have proved case (ii), this finishes the case $l(\mathcal{R})=1$.

Next, suppose $l(\mathcal{R})>1$. In this case, Lemma 4.9(iii)(4) implies

$$
\operatorname{deg}_{C_{b-1}} \mathcal{R}_{2, j}=-1 \text {. }
$$

Set $\Psi^{\prime}=T_{\mathcal{O}_{C_{b}}(-1)} \circ T_{\mathcal{O}_{C_{b-1}}(-2)}$. Then we have

$$
\begin{aligned}
& \Psi^{\prime}(\alpha) \cong \mathcal{O}_{C_{b-1}}(-2) \quad \text { and } \quad l\left(\Psi^{\prime}(\beta)\right) \leqslant l(\beta) \\
& C_{b-2} \quad C_{b-1} \quad C_{b} \quad C_{b+1}
\end{aligned}
$$

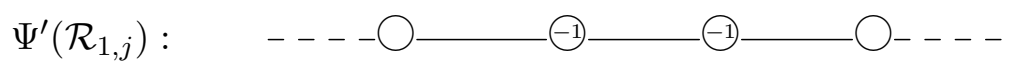

$$
\begin{aligned}
& \Psi^{\prime}\left(\mathcal{R}_{2, j}\right) \text { : } \\
& \Psi^{\prime}\left(\mathcal{R}_{3}\right) \\
& ---0^{-} \\
& (-1)-\bigcirc_{-}--\quad: \Psi^{\prime}\left(\mathcal{R}_{4}\right) \\
& \Psi^{\prime}(\alpha) \text { : }
\end{aligned}
$$

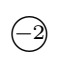

First, let us consider the case $C_{s(\mathcal{R})}=C_{b+1}$ or, equivalently, $\mathcal{R}=\mathcal{R}^{\prime}$. Take an element $\widetilde{\mathcal{R}_{2, j}} \in \Sigma(\beta)$ such that $\left.\widetilde{\mathcal{R}_{2, j}}\right|_{U_{b-1, b}}$ contains $\mathcal{R}_{2, j}$ as a direct summand. Then $\Psi^{\prime}\left(\widetilde{\mathcal{R}_{2, j}}\right)$ satisfies

$$
t\left(\Psi^{\prime}\left(\widetilde{\mathcal{R}_{2, j}}\right)\right)-n+1<b-1<s\left(\Psi^{\prime}\left(\widetilde{\mathcal{R}_{2, j}}\right)\right)-1
$$

or

$$
t\left(\Psi^{\prime}\left(\widetilde{\mathcal{R}_{2, j}}\right)\right)+1<b-1 .
$$

Hence, we can find $\Psi$ as in Claim 6.1 , by Remark 6.8. Therefore, we may assume $C_{s(\mathcal{R})} \neq C_{b+1}$. Note that $\Psi^{\prime}\left(H^{q}(\beta)\right)$ is a sheaf for every $q \in \mathbb{Z}$. Using the spectral sequence

$$
E_{2}^{p, q}=H^{p}\left(\Psi^{\prime}\left(H^{q}(\beta)\right)\right) \Longrightarrow E^{p+q}=H^{p+q}\left(\Psi^{\prime}(\beta)\right),
$$

we see that $\Psi^{\prime}\left(H^{q}(\beta)\right)=H^{q}\left(\Psi^{\prime}(\beta)\right)$, and then $\left(\Psi^{\prime}(\alpha), \Psi^{\prime}(\beta)\right)$ fits in case (iii). Furthermore, the assumption $C_{s(\mathcal{R})} \neq C_{b+1}$ implies $l\left(\Psi^{\prime}(\mathcal{R})\right)<l(\mathcal{R})$. Hence, we can conclude by induction on $l(\mathcal{R})$.

\section{Example}

If $\lambda_{S} \leqslant 4$, we have $H_{S}=\left(\mathbb{Z} / \lambda_{S} \mathbb{Z}\right)^{*}$ and $\operatorname{Im} \Theta=\Gamma_{0}\left(\lambda_{S}\right)$, as pointed out in Remark 3.12. For a general elliptic surface $S$, however, it is not easy to describe the group $H_{S}$ concretely. In this section, we give an example of an elliptic surface with $\lambda_{S} \geqslant 5$ where we are able to determine $H_{S}$, and hence $\operatorname{Im} \Theta$, more concretely.

Let us consider a rational elliptic surface $\pi: J \rightarrow \mathbb{P}^{1}$ with a section, and assume that $\pi$ has four singular fibers of types $\mathrm{I}_{7}, \mathrm{I}_{2}$, II and $\mathrm{I}_{1}$. Such a surface exists by Persson's list [Per90]. Take a point $s \in \mathbb{P}^{1}$ over which the fiber of $\pi$ is smooth or of type $\mathrm{I}_{1}$. Apply a logarithmic transformation along the point $s$ to obtain a rational elliptic surface $S$ with Jacobian surface $J$ and a multiple fiber of multiplicity $m$ over the point $s$. Suppose $m>2$. Then, as in [Ueh12, Example 2.6], we can show $H_{S}=\{ \pm 1\}$ (we leave it to the reader to check this). Therefore, 


\section{H. UEHARA}

Theorem 4.1 assures that there is a short exact sequence:

$$
\begin{aligned}
1 & \rightarrow\left\langle B, \otimes \mathcal{O}_{S}(D) \mid D \cdot F=0\right\rangle \rtimes \operatorname{Aut} S \times \mathbb{Z}[2] \rightarrow \operatorname{Auteq} D(S) \stackrel{\Theta}{\rightarrow} \\
& \stackrel{\Theta}{\rightarrow}\left\{\left(\begin{array}{ll}
c & a \\
d & b
\end{array}\right) \in \Gamma_{0}(m) \mid b \equiv \pm 1(\bmod m)\right\} \rightarrow 1 .
\end{aligned}
$$

In this case, Aut $S=\operatorname{Aut}_{\mathbb{P}^{1}} S$ is just the semi-direct product of the Mordell-Weil group of $S$ and the subgroup of automorphisms preserving the zero section (cf. [FM94, Theorem 1.3.14]). Hence, it can be calculated by using [OS91].

In [Ueh15], the author considers the autoequivalence group and Fourier-Mukai partners of elliptic ruled surfaces.

\section{ACKNOWLEDGEMENTS}

This paper was written during the author's staying at the Max Planck Institute for Mathematics, in the period from April to September 2014. The author appreciates the hospitality. He also thanks Yukinobu Toda for pointing out some mistakes in the first draft.

\section{REFERENCES}

Ati57 M. F. Atiyah, Vector bundles over an elliptic curve, Proc. London Math. Soc. 7 (1957), 414-452; http://dx.doi.org/10.1112/plms/s3-7.1.414.

BBDG06 L. Bodnarchuk, I. Burban, Yu. Drozd and G.-M. Greuel, Vector bundles and torsion free sheaves on degenerations of elliptic curves, in Global Aspects of Complex Geometry (Springer, Berlin, 2006), 83-128; http://dx.doi.org/10.1007/3-540-35480-8_3.

Bea96 A. Beauville, Complex algebraic surfaces, 2nd ed., London Math. Soc. Stud. Texts, vol. 34 (Cambridge University Press Cambridge, 1996); http://dx.doi.org/10.1017/CB09780511623936.

BHPV04 W.P. Barth, K. Hulek, C. A. M. Peters and A. van de Ven, Compact complex surfaces, 2nd ed., Ergeb. Math. Grenzgeb. (3), vol. 4 (Springer-Verlag, Berlin, 2004); http://dx.doi.org/10. 1007/978-3-642-57739-0.

BM98 T. Bridgeland and A. Maciocia, Fourier-Mukai transforms for quotient varieties, 1998, arXiv:math.AG/9811101.

BM01_ Complex surfaces with equivalent derived categories, Math. Z. 236 (2001), no. 4, 677697; http://dx.doi.org/10.1007/PL00004847.

BM02 - Fourier-Mukai transforms for K3 and elliptic fibrations, J. Algebraic Geom. 11 (2002), no. 4, 629-657; http://dx.doi.org/10.1090/S1056-3911-02-00317-X.

BO97 A.I. Bondal and D.O. Orlov, Semiorthogonal decomposition for algebraic varieties, 1997, arXiv:alg-geom/9712029.

Bri98 T. Bridgeland, Fourier-Mukai transforms for elliptic surfaces, J. reine angew. Math. 498 (1998), 115-133; http://dx.doi.org/10.1515/crll.1998.046.

Bri99_L Equivalences of triangulated categories and Fourier-Mukai transforms, Bull. London Math. Soc. 31 (1999), no. 1, 25-34; http://dx.doi.org/10.1112/S0024609398004998.

BV03 A. Bondal and M. Van den Bergh, Generators and representability of functors in commutative and noncommutative geometry, Mosc. Math. J. 3 (2003), no. 1, 1-36.

FM94 R. Friedman and J.W. Morgan, Smooth four-manifolds and complex surfaces, Ergeb. Math. Grenzgeb. (3), vol. 27 (Springer-Verlag, Berlin, 1994); http://dx.doi.org/10.1007/ 978-3-662-03028-8.

Fri95 R. Friedman, Vector bundles and $\mathrm{SO}(3)$-invariants for elliptic surfaces, J. Amer. Math. Soc. 8 (1995), no. 1, 29-139; http://dx.doi.org/10.2307/2152883. 


\section{Autoequivalences of Elliptic surfaces}

Har77 R. Hartshorne, Algebraic geometry, Grad. Texts in Math., vol. 52 (Springer-Verlag, New YorkHeidelberg, 1977); http://dx.doi.org/10.1007/978-1-4757-3849-0.

HLS09 D. Hernández Ruipérez, A. C. López Martín and F. Sancho de Salas, Relative integral functors for singular fibrations and singular partners, J. Eur. Math. Soc. (JEMS) 11 (2009), no. 3, 597-625; http://dx.doi.org/10.4171/JEMS/162.

Huy06 D. Huybrechts, Fourier-Mukai transforms in algebraic geometry, Oxford Math. Monogr. (The Clarendon Press, Oxford Univ. Press, Oxford, 2006); http://dx.doi.org/10.1093/acprof: oso/9780199296866.001.0001.

IU05 A. Ishii and H. Uehara, Autoequivalences of derived categories on the minimal resolutions of $A_{n}$-singularities on surfaces, J. Differential Geom. 71 (2005), no. 3, 385-435; http: //projecteuclid.org/euclid.jdg/1143571989.

IUU10 A. Ishii, K. Ueda and H. Uehara, Stability conditions on $A_{n}$-singularities, J. Differential Geom. 84 (2010), no. 1; 87-126, http://projecteuclid.org/euclid.jdg/1271271794.

Kaw02 Y. Kawamata, D-equivalence and K-equivalence, J. Differential Geom. 61 (2002), no. 1, 147171; http://projecteuclid.org/euclid.jdg/1090351323.

KO94 S.A. Kuleshov and D. O. Orlov, Exceptional sheaves on Del Pezzo surfaces, Russian Acad. Sci. Izv. Math. 58 (1994), no. 3, 479-513; http://dx.doi.org/10.1070/ IM1995v044n03ABEH001609.

LST13 A. C. López Martín, D. Sánchez Gómez and C. Tejero Prieto, Relative Fourier-Mukai transforms for Weierstraß fibrations, abelian schemes and Fano fibrations, Math. Proc. Cambridge Philos. Soc. 155 (2013), no. 1, 129-153; http://dx.doi.org/10.1017/S0305004113000029.

Orl97 D. O. Orlov, Equivalences of derived categories and K3 surfaces, J. Math. Sci. 84 (1997), no. 5, 1361-1381; http://dx.doi.org/10.1007/BF02399195.

Orl02 Derived categories of coherent sheaves on abelian varieties and equivalences between them, Izv. Math. 66 (2002), no. 3, 569-594; http://dx.doi.org/10.1070/ IM2002v066n03ABEH000389.

OS91 K. Oguiso and T. Shioda, The Mordell-Weil lattice of a rational elliptic surface, Comment. Math. Univ. St. Pauli 40 (1991), no. 1, 83-99; http://dx.doi.org/10.14992/00009974.

Per90 U. Persson, Configurations of Kodaira fibers on rational elliptic surfaces, Math. Z. 205 (1990), no. 1, 1-47; http://dx.doi.org/10.1007/BF02571223.

ST01 P. Seidel and R. Thomas, Braid group actions on derived categories of coherent sheaves, Duke Math. J. 108 (2001), no. 1, 37-108; http://dx.doi.org/10.1215/S0012-7094-01-10812-0.

Tod06 Y. Toda, Fourier-Mukai transforms and canonical divisors, Compos. Math. 142 (2006), no. 4, 962-982; http://dx.doi.org/10.1112/S0010437X06001977.

Ueh04 H. Uehara, An example of Fourier-Mukai partners of minimal elliptic surfaces, Math. Res. Lett. 11 (2004), no. 2-3, 371-375; http://dx.doi.org/10.4310/MRL.2004.v11.n3.a9.

Ueh12 , A counterexample of the birational Torelli problem via Fourier-Mukai transforms, J. Algebraic Geom. 21 (2012), no. 1, 77-96; http://dx.doi.org/10.1090/ S1056-3911-2011-00551-1.

Ueh15_ Fourier-Mukai partners of elliptic ruled surfaces, Proc. Amer. Math. Soc., to appear, arXiv:1511.06031.

Hokuto Uehara hokuto@tmu.ac.jp

Department of Mathematics and Information Sciences, Tokyo Metropolitan University, 1-1 Minamiohsawa, Hachioji-shi, Tokyo, 192-0397, Japan 